\title{
Ethnobotanique du cocotier (Cocos nucifera L.) sur l'île de Vanua Lava (Vanuatu)
}

\section{Sophie Caillon}

\section{(2) OpenEdition}

12 Journals

Édition électronique

URL : http://journals.openedition.org/jso/6533

DOI : $10.4000 /$ jso.6533

ISSN : $1760-7256$

Éditeur

Société des océanistes

\section{Édition imprimée}

Date de publication : 15 décembre 2011

Pagination : 333-352

ISBN : 978-2-85430-037-4

ISSN : 0300-953x

\section{Référence électronique}

Sophie Caillon, «Ethnobotanique du cocotier (Cocos nucifera L.) sur l'île de Vanua Lava (Vanuatu) », Journal de la Société des Océanistes [En ligne], 133 | 2e semestre 2011, mis en ligne le 31 décembre 2014, consulté le 10 décembre 2020. URL : http://journals.openedition.org/jso/6533 ; DOI : https:// doi.org/10.4000/jso.6533

\section{(c) $(1) \ominus$}

Journal de la société des océanistes est mis à disposition selon les termes de la Licence Creative Commons Attribution - Pas d'Utilisation Commerciale - Pas de Modification 4.0 International. 


\section{Ethnobotanique du cocotier (Cocos nucifera L.) sur l'île de Vanua Lava (Vanuatu)}

par

Sophie CAILLON*

\section{RÉSUMÉ}

Depuis la fin du XIXe siècle et jusqu'à nos jours, l'économie du Vanuatu est largement dépendante de l'industrie du coprah. L'extension des cocoteraies sur les rivages insulaires est une des évolutions les plus visibles causées par le développement de cette industrie. Même si les cocotiers étaient présents sur les îles avant l'installation des premiers hommes, la colonisation a transformé l'identité socioculturelle du cocotier. Dans cet article, nous traitons de l'ethnobotanique du cocotier dans le village de Vētuboso sur l'île de Vanua Lava (groupe des Banks). L'évolution de son système de classification et de nomenclature, de la diversité de ses usages (techniques et culturels) et de son rôle symbolique (mythes et histoires associés, anthropomorphisme) porte témoignage de cette histoire. Aujourd'hui, certains habitants de Vanua Lava l'appellent "l'arbre des Blancs".

MoTS-ClEFS : anthropomorphisme, alimentation, classification, mythe, nomenclature, Mélanésie, usages

Plus de trente ans après l'indépendance du Vanuatu, la marque la plus visible du temps colonial est la transformation des paysages par l'extension des cocoteraies sur un espace insulaire restreint (Labouisse, 2004). Ces nouvelles plantations symbolisent la transition des habitants du pays entre le monde des hommes-lieux, man ples, et celui des hommes blancs, entre les mondes de la kastom et de la skul (Bonnemaison, 1996 : 255). L'organisation des plantations au Vanuatu est donc le reflet d'un passé colonial et d'un présent engagé dans une économie monétaire de plus en plus globale.

\begin{abstract}
Since the end of the $19^{\text {th }}$ century, the economy of Vanuatu is mainly based on copra exportation. One of the most obvious changes can be observed on the coasts of these islands through the spread of coconut plantations. Even if coconuts were present before the arrival of the first human being, copra industry has modified the sociocultural identity of the coconut. In this article, we analyse the ethnobotany of the coconut in the village of Vétuboso on the island of Vanua Lava (Banks group). The evolution of its system of classification and nomenclature, of the diversity of its uses (technical and cultral) and of its symbolic role (myths and stories, anthropomorphism) attests of this history. Nowadays, even few inhabitants from Vanua Lava name it the "tree from the Whites".
\end{abstract}

KEYwORDS: anthropomorphism, classification, food, Melanesia, myth, nomenclature, uses

Avec les autres arbres à noix, comme les nangaillers ( $\overline{\boldsymbol{n}}$, Canarium indicum L. ou C. harveyi Seeman) et les velliers (wōtag, les Barringtonia edulis J.R. Forst. \& G. Forst.), les cocotiers étaient auparavant plantés le long des sentiers, autour des jardins ou des tarodières afin d'apaiser la faim des travailleurs et préparer des spécialités culinaires. Avec l'émergence d'une économie du coprah, le cocotier a été " mis en culture " dans un nouvel espace, la cocoteraie. Alors que le cocotier changeait d'espace - des sentiers aux plantations monospécifiques - le nombre d'arbres plantés augmenta. Les agriculteurs des îles eurent

\footnotetext{
Chargée de recherche au CNRS, UMR 5175 CEFE Équipe interactions bioculturelles, sophie.caillon@cefe.cnrs.fr
} 


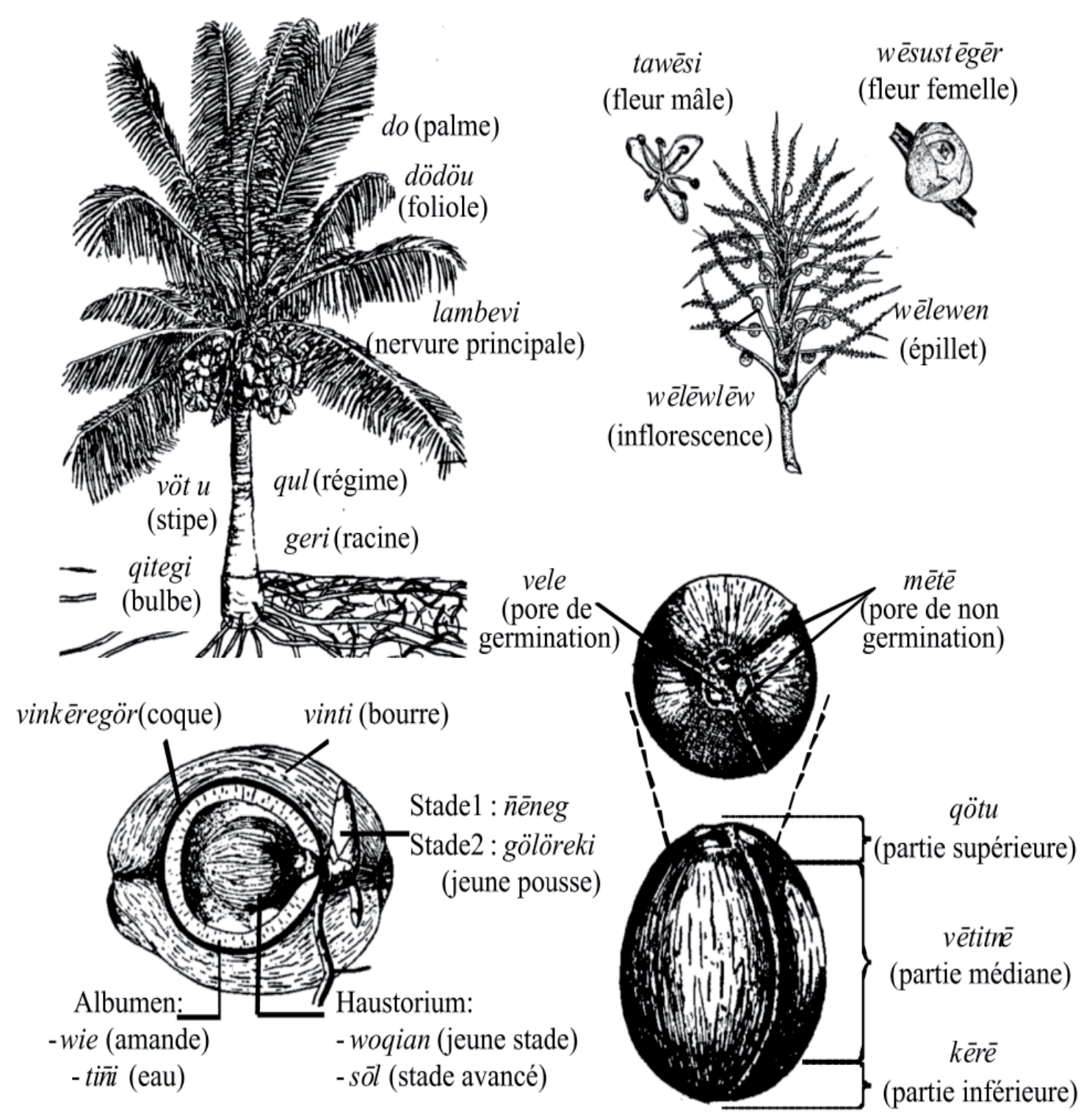

Figure I - Description morphologique du cocotier en vurës (dessins de Taffin, 1993)

besoin d'un grand nombre de cocotiers pour satisfaire leurs nouveaux besoins monétaires en vendant du coprah. Ils allèrent chercher des semences dans les plantations coloniales proches dont le matériel biologique avait été introduit d'autres îles. La multiplication des cocotiers, l'introduction en masse d'arbres allochtones et le changement des critères de sélection privilégiant de larges fruits à forte teneur en chair, contribuèrent à modifier les caractères morphologiques des cocotiers de l'île.

Pour assurer des fonctions opératoires de l'ordre du cognitif comme identifier, mémoriser et communiquer sur des végétaux impliqués dans des usages techniques ou culturels, ou ayant une fonction écologique, l'homme cherche les discontinuités afin d'extraire des unités percep- tuelles élémentaires ; discriminer, catégoriser et nommer font partie des fondamentaux (Friedberg, 1997). Dans cet article, nous nous intéressons à la manière dont le changement culturel matérialisé ici par l'évolution du traitement du cocotier dans la société vanuataise, influence le système de classification, de nomenclature et les usages du cocotier. Quelle est sa position classificatoire? Suivant quelle logique les planteurs nomment-ils les anciennes et nouvelles formes de cocotiers? Comment et sur quels fondements ont-ils choisi les critères discriminants et les ontils hiérarchisés ?

Des observations ethnobotaniques sur le cocotier ont été réalisées dans un village situé à l'ouest de Vanua Lava, la plus grande île $\left(331 \mathrm{~km}^{2}\right.$ et 1933 habitants en 2001) du groupe des Banks, 


\begin{tabular}{|c|c|c|}
\hline $\mathbf{N}^{\circ}$ & Nom du stade & Description des organes marquant chaque stade de croissance \\
\hline 1 & $s \bar{o} l$ & Terme général pour désigner les jeunes fruits germés \\
\hline 2 & woqian & Cocotier dont les fruits germés ont un petit haustorium \\
\hline 3 & gölörēki & Cocotier dont les fruits germés sont surmontés de jeunes palmes \\
\hline 4 & bar & Cocotier dont les fruits germés ne contiennent plus d'haustorium \\
\hline 5 & rōwsiag & Le cocotier est bien enraciné \\
\hline 6 & $\bar{o} w$ or sigeg qötū̄w & Cocotier dont la première spathe émerge \\
\hline 7 & webeges & Cocotier dont la première spathe s'ouvre \\
\hline 8 & tawēsi & Cocotier dont la première fleur éclot \\
\hline 9 & wēsustēgēr & Cocotier dont les fruits ne contiennent toujours pas d'eau \\
\hline 10 & $m \ddot{e}$ & Cocotier dont les fruits contiennent de l'eau mais pas d'amande \\
\hline 11 & vōsgargarteqërët & $\begin{array}{l}\text { Cocotier dont les fruits à coque fragile contiennent de l'eau et une } \\
\text { fine couche d'amande gélatineuse }\end{array}$ \\
\hline 12 & $v \overline{o s}$ & $\begin{array}{l}\text { Cocotier dont les fruits à coque solide contiennent de l'eau et de } \\
\text { l'amande gélatineuse }\end{array}$ \\
\hline 13 & mian /qōtō & Cocotier dont les fruits presque secs contiennent de l'air \\
\hline 14 & gēbibiag & Cocotier dont l'amande est sèche mais l'épiderme est toujours vert. \\
\hline 15 & mereñ or matabōbōt & Cocotier dont les fruits sont secs et encore sur l'arbre (merē̄, sec) \\
\hline 16 & solqetqet ou & Cocotier dont les fruits germés sont coincés dans l'arbre \\
\hline 17 & mës & Cocotier dont les fruits secs viennent de tomber de l'arbre \\
\hline 18 & 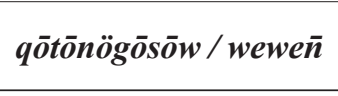 & $\begin{array}{l}\text { Cocotier dont le fruit ne germe pas et dont la noix est vide après la } \\
\text { décomposition de la bourre et de l'amande }\end{array}$ \\
\hline
\end{tabular}

TABlEAU I - Les dix-huit stades de croissance du cocotier en vurës

un archipel situé au nord du Vanuatu. Le village de Vētuboso est le principal village de l'île avec 610 habitants en 2003 qui parlent le vurës ${ }^{1}$. Les unités de base des groupes de filiation de Vètu-

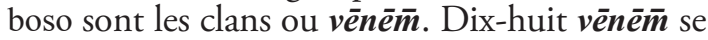
répartissent en deux moitiés exogames non nommées. Le système est matrilinéaire et patrilocal (Hess, 2009).

Des discussions informelles et des enquêtes semi-directives auprès de 24 chefs de famille entre 35 et 75 ans sur les lieux d'habitation puis sur les plantations ont alimenté l'étude ethnobotanique. Discussions et enquêtes portaient sur l'usage, les besoins agronomiques, les modalités de mise en culture de chaque catégorie nommée (ensemble des individus d'une espèce donnée pour lesquels un groupe de locuteurs d'une même langue s'accordent pour attribuer un nom unique). Un relevé exhaustif du nombre d'arbres par catégorie de cocotiers a été réalisé dans 19 plantations pendant une période de six mois répartie sur deux ans, entre 2001 et 2003. Des questionnaires fermés auprès de 56 informateurs entre 18 et 73 ans dont 28 femmes portaient sur des données socio-économiques (scolarisation, nombre d'enfants, de plantations, de fours à coprah, etc.) et sur leur connaissance des 24 principales catégories nommées ( $c f$. colonne Enquêté du tableau 2). La présence ou non de ces catégories dans leurs plantations a aussi été notée. Les analyses statistiques ont été réalisées sous R (R-Development-Core-Team, 2008). Une autre série de questionnaires a été remplie par 40 personnes réparties sur 19 îles du Vanuatu, afin d'inventorier les noms des cocotiers et leurs histoires associées. Pour évaluer et tester la manière dont les habitants de Vêtuboso apprécient les qualités gustatives des différentes catégories nommées, nous avons organisé des tests organoleptiques (épreuves de classement et triangulaire) avec un jury de 30 personnes (entre 16 et 82 ans, dont $50 \%$ de femmes).

Après l'étude du système de classification et de nomenclature du cocotier, nous nous intéressons aux usages quotidiens puis cérémoniels, médicinaux et magiques. Les attributs anthropomorphiques du cocotier complètent cette description ethnobotanique du cocotier.

1. Les termes dans la langue vurës ou autres langues vernaculaires sont notés en gras et italiques. Cette langue s'écrit à l'aide de 15 consonnes et 10 voyelles (Malau, 2011). Ceux en bichlamar, la langue véhiculaire du Vanuatu, et les noms scientifiques des plantes et des animaux sont écrits en italiques. Les noms des catégories nommées de cocotier sont en gras. 


\begin{tabular}{|c|c|c|c|c|}
\hline Code & $\begin{array}{l}\text { Nom catégorie en } \\
\text { vurës }\end{array}$ & Traduction & Description donnée par les planteurs & Enquêté \\
\hline 1 & mōtō malgias & $\begin{array}{l}\text { malgias milieu, ni vert ni } \\
\text { rouge }\end{array}$ & Fruits « ni rouges, ni verts » (kaki) & $\mathrm{X}$ \\
\hline 2 & mōtō mamē & gamamē rouge & Fruits rouges & $\mathrm{X}$ \\
\hline 3 & mōtō gōtōtōōōg & gōtōtōrōg vert & Fruits verts & $\mathrm{X}$ \\
\hline 4 & $\begin{array}{l}\text { mōtō } \\
\text { gōtōtōrōgqȫ̄̄qön }\end{array}$ & gōtōtōōōg vert ; qȫn foncé & Fruits verts foncés & \\
\hline 5 & mōtō wulmēe & & Anneau rouge autour de l'œil de germination & $\mathrm{X}$ \\
\hline 6 & mōtō gaañā̄ & gaañañ jaune & $\begin{array}{l}\text { "Albinos " (fruits, inflorescence et palmes } \\
\text { jaunes) }\end{array}$ & $\mathrm{X}$ \\
\hline 7 & sōgsōg & & Petits fruits nombreux & $\mathrm{X}$ \\
\hline 8 & mōtō geluwō & geluwō gros & Gros fruits et grosses noix & $\mathrm{X}$ \\
\hline 9 & mōtō vingaqō & vin bourre ; gaqō épaisse & Gros fruits et petites noix (longues fibres) & $\mathrm{X}$ \\
\hline 10 & mōtō gemetestes & testes pointu & Fruits petits, allongés et pointus & $\mathrm{X}$ \\
\hline 11 & mōtō wesum̄olo & $\begin{array}{l}\text { wesu } \text { œuf; } \overline{\boldsymbol{m}} \text { olo type de } \\
\text { canard }\end{array}$ & Fruits en forme d'œuf & \\
\hline 12 & mōtō meteröwö & röwö type de poisson & Noix ressemblant à une tête de poisson & $\mathrm{X}$ \\
\hline 13 & mōtō seseser & seseser tirer (ex. cheveux) & Bourre soft (se retire à la main) & $\mathrm{X}$ \\
\hline 14 & mōtō dēdērēs & dēèēēs sucré & Amande et eau sucrées & $\mathrm{X}$ \\
\hline 15 & mōtō us & & Amande juteuse et sucrée & $\mathrm{X}$ \\
\hline 16 & mōtō mölumlum & mölumlum mou & Amande molle & $\mathrm{X}$ \\
\hline 17 & mōtō gagrak & gagrak séparer, détacher & Amande facilement extractible & $\mathrm{X}$ \\
\hline 18 & mōtō vet & ve, pierre & $\begin{array}{l}\text { Amande dure dont on extrait beaucoup } \\
\text { d'huile }\end{array}$ & $\mathrm{X}$ \\
\hline 19 & mōtō silat & $\begin{array}{l}\text { silat l'arbre Dendrocnide } \\
\text { latifolia }\end{array}$ & Palmes fragiles (se détachent facilement) & $\mathrm{X}$ \\
\hline 20 & mōtō lak & lak danser & $\begin{array}{l}\text { Palmes en mouvement continuel (qui « dan- } \\
\text { sent })\end{array}$ & \\
\hline 21 & mōtō tak(tak) & $\begin{array}{l}\text { taktak quand une pierre est } \\
\text { jetée, les fruits tombent }\end{array}$ & Forme spicata (inflorescence sans épillets) & $\mathrm{X}$ \\
\hline 22 & mōtō reqe & reqe femme & Petit cocotier avec gros fruits & $\mathrm{X}$ \\
\hline 23 & mōtō mētigtisēe & mētigtisēé égoïste & Peu de fruits & \\
\hline 24 & mōtō at̄̄̄ēn & $\boldsymbol{a t} \overline{\boldsymbol{m}} \overline{\boldsymbol{e}} \boldsymbol{n}$ homme & Long cocotier avec peu de fruits & $\mathrm{X}$ \\
\hline 25 & mōtō $\bar{m} a t$ & $\overline{\mathbf{m}} \boldsymbol{a t}$ serpent & Stipe en spirale & $\mathrm{X}$ \\
\hline 26 & mōtō varam & varam jumeaux & Deux cocotiers ont germé d'un fruit & \\
\hline 27 & mōtō bal & $\begin{array}{l}\text { gabal pince pour enlever les } \\
\text { pierres chaudes du four }\end{array}$ & Deux cocotiers plantés l'un à côté de l'autre & $\mathrm{X}$ \\
\hline 28 & mōtō sialmē & sial flotter ; vanmē arriver & Cocotier venu de la mer & $\mathrm{X}$ \\
\hline 29 & mōtō qet & Qet héros mythique des Banks & Cocotier du héros mythique Qet & \\
\hline 30 & mōtō vanvan & van aller se promener & Cocotier qui ne produit que 6 mois & $\mathrm{X}$ \\
\hline 31 & mōtō wewese & wewese rond & Fruits ronds & \\
\hline 32 & $\begin{array}{l}\text { mōtō } \\
\text { wösusū̄almal }\end{array}$ & 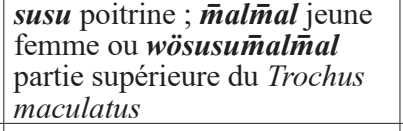 & Fruits ronds avec une pointe & \\
\hline 33 & mōtō mēvinvin & mēvinvin fin & Coque fine & \\
\hline 34 & mōtō mötöltöl & mötöltöl épais & Coque épaisse & \\
\hline 35 & mōtō sörsör & & Cocotier sans fruits & \\
\hline 36 & mōtō lenman & lenman strié & Fruits striés de deux couleurs & \\
\hline 37 & mōtō sergabē & gabē eau & Bourre solide et imperméable & \\
\hline 38 & mōtō lēnötō & $\boldsymbol{l} \overline{\boldsymbol{e}}$ crēte $; \boldsymbol{n} \ddot{\boldsymbol{o}}$ de $; \boldsymbol{t} \overline{\boldsymbol{o}}$ poule & Extrémités des épillets aplatis et dentelés & \\
\hline
\end{tabular}

Le nom est traduit et le cocotier portant ce nom est décrit. Par une croix, les cocotiers ayant fait l'objet d'un contrôle de connaissance auprès de 56 personnes sont signalés (colonne enquêté).

TABleau 2 - Liste des trente-huit catégories nommées de cocotiers de Vanua Lava en vurës 


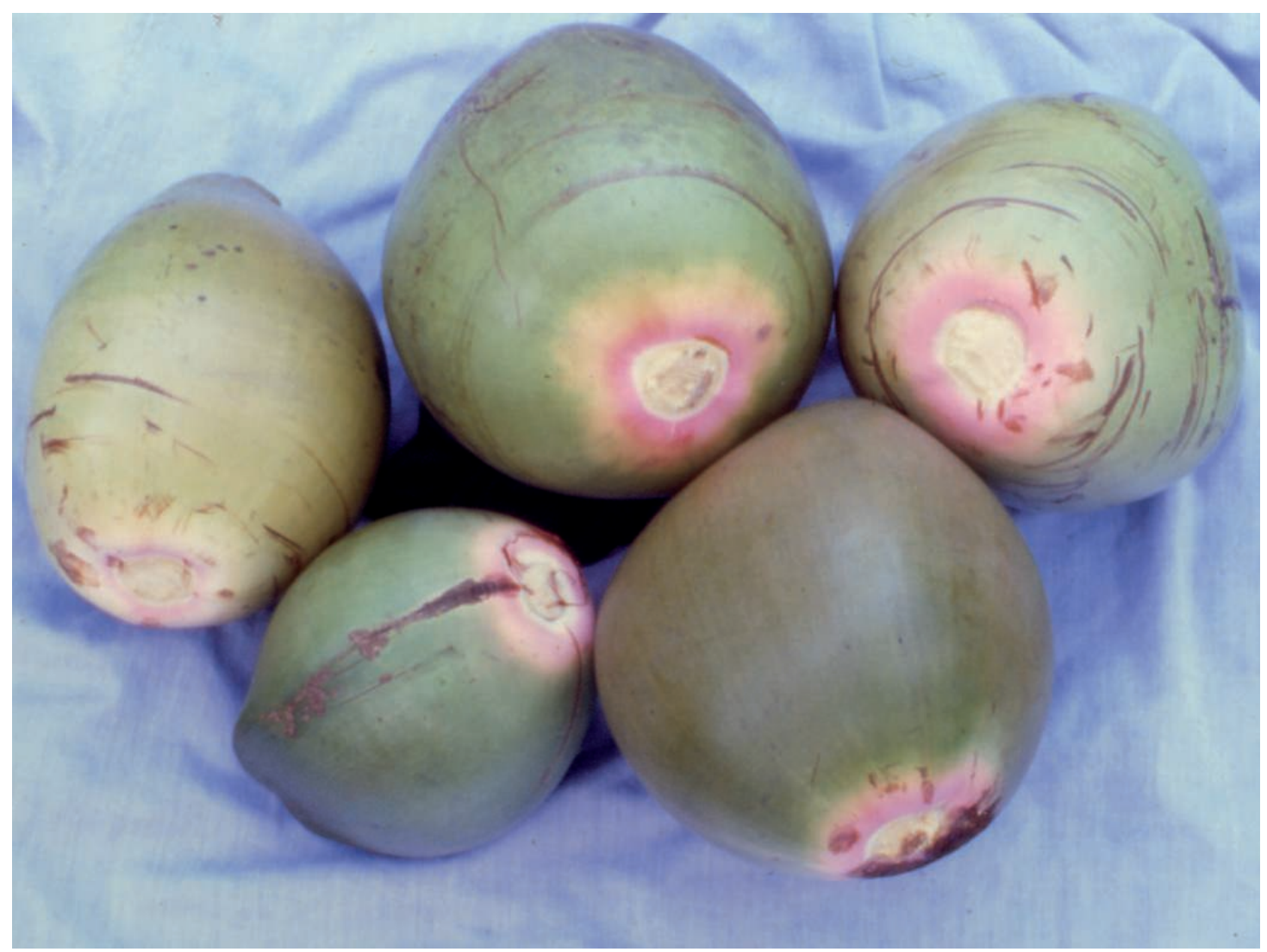

Рното 1. - Catégorie nommé $\boldsymbol{m} \overline{\boldsymbol{o}} \boldsymbol{t} \boldsymbol{o}$ wulmēe, caractérisée par un anneau rose-rouge autour de l'œil de germination (Vētuboso, Vanua Lava, 2002, cliché de l'auteur)

Système de classification et de nomenclature du cocotier

\section{Classer : un arbre à la frontière du domestique et} du sauvage

À Vētuboso, le cocotier est classé au sein de la catégorie englobante lövlöv, le monde du «bois » avec les autres plantes pérennes dont le tronc (ou stipe dans le cas des palmiers) est dur. Un arbre individuel est appelé en vurës rēentenge. La nomenclature locale décrit précisément l'ensemble de ses organes végétatifs et de reproduction (fig. 1). Les parties mâles et femelles sont différenciées, et l'existence d'une fécondation croisée entre individus distincts est connue. Les agriculteurs savent que les abeilles et le vent sont des moyens de dispersion du pollen. Ils assimilent l'amande de la noix à une graine et la bourre à la pulpe d'un fruit. Ainsi un fruit de cocotier sans amande est appelé wamarbō, un terme réservé aux cocotiers pour décrire un fruit vide de graine n'engendrant pas la vie. Les habitants de Vētuboso reconnaissent dix-huit stades de croissance du cocotier (tab. 1). Quatorze décrivent l'évolution de la taille, de la couleur et de la consistance des différents éléments du fruit, trois le développement de l'inflorescence et un seul (rōwsiag) est réservé à l'arbre dans son ensemble.
Au cours de son histoire, le statut du cocotier a évolué passant de celui de plante spontanée à celui de plante encouragée puis domestiquée (Harlan, 1987[1975] : 75). Il est apparu naturellement sur les rivages des îles du Pacifique grâce à la capacité de dispersion par flottaison de ses semences. Sa multiplication fut très vite encouragée par les populations qui s'installèrent sur ces îles isolées, en particulier celles dépourvues de rivières. Il fut aussi amené dans les pirogues des premiers migrants austronésiens, puis devient un objet d'échanges entre les populations des îles.

Issu de deux parents différents, le cocotier vit le temps d'une vie humaine (80 ans), soit trois à quatre générations d'homme. Non seulement le temps de génération limite la compréhension de son évolution sur plusieurs générations, mais son mode de reproduction par fécondation croisée à des hauteurs peu accessibles, n'autorise pas l'homme à maîtriser l'identité de la descendance et donc la nature de ce qu'il plante. En échappant au contrôle de l'homme, que ce soit pour son introduction ou pour sa multiplication, le cocotier est un être indépendant, et malgré sa plantation en ligne dans des espaces anthropisés monospécifiques, il conserve son statut d'arbre fruitier. Arbre intermédiaire entre le cultivé et le spontané, entre le milieu domestique et sauvage, 


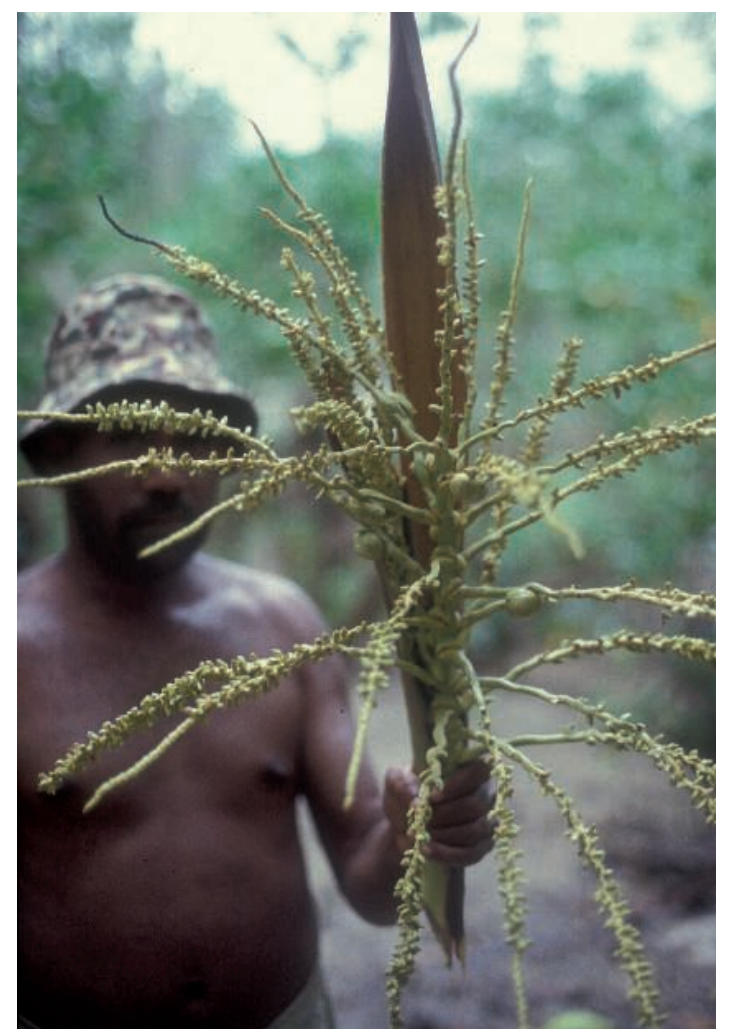

Рното 2. - Inflorescence sans épillet du cocotier de forme spicata, localement nommé mōtō taktak (Vētuboso, Vanua Lava, 2002, cliché de l'auteur) le cocotier vit entre deux espaces, ceux du village et de la forêt où habitent respectivement les vivants et les morts qui rôdent (timiat $\left.{ }^{2}\right)$.

\section{Nommer connaître et cultiver la diversité à Vanua Lava}

Dans le village de Vētuboso, et plus largement sur la côte ouest de Vanua Lava (villages de Kērēbētia, Wasag et Vatrata), trente-huit noms de cocotiers ont pu être inventoriés (tab. 2) et constituent autant de catégories nommées. La synthèse de plusieurs sources de données (Labouisse and Caillon, 2001; Caillon, 2004) ${ }^{3}$ a permis de recenser soixante-quinze catégories nommées de cocotiers à l'échelle du Vanuatu, avec une moyenne de 22 par site (minimum : 4 à Marino au nord de Maewo ; maximum : 73 à Levravuh Bay sur Malakula). Les îles réputées pour leurs vastes cocoteraies - Malekula (village de Levravuh Bay), Tanna (Latadu White Sand), Gaua (Qwetevut), Ambrym (Faula), Santo (Tassiriki) et Pentecôte (Loltong) - comptent chacune plus de trente-neuf catégories nommées.

Les critères d'identification et les règles de nomenclature sont indissociables à Vetuboso : le terme de base (ou niveau de base) " cocotier " $\boldsymbol{m} \overline{\boldsymbol{o}} \boldsymbol{t} \overline{\boldsymbol{o}}$ est, dans 37 cas, toujours placé en tête et prend une valeur de "type " (Grenand, 20012002 : 22) ; il est suivi par un déterminant qua-

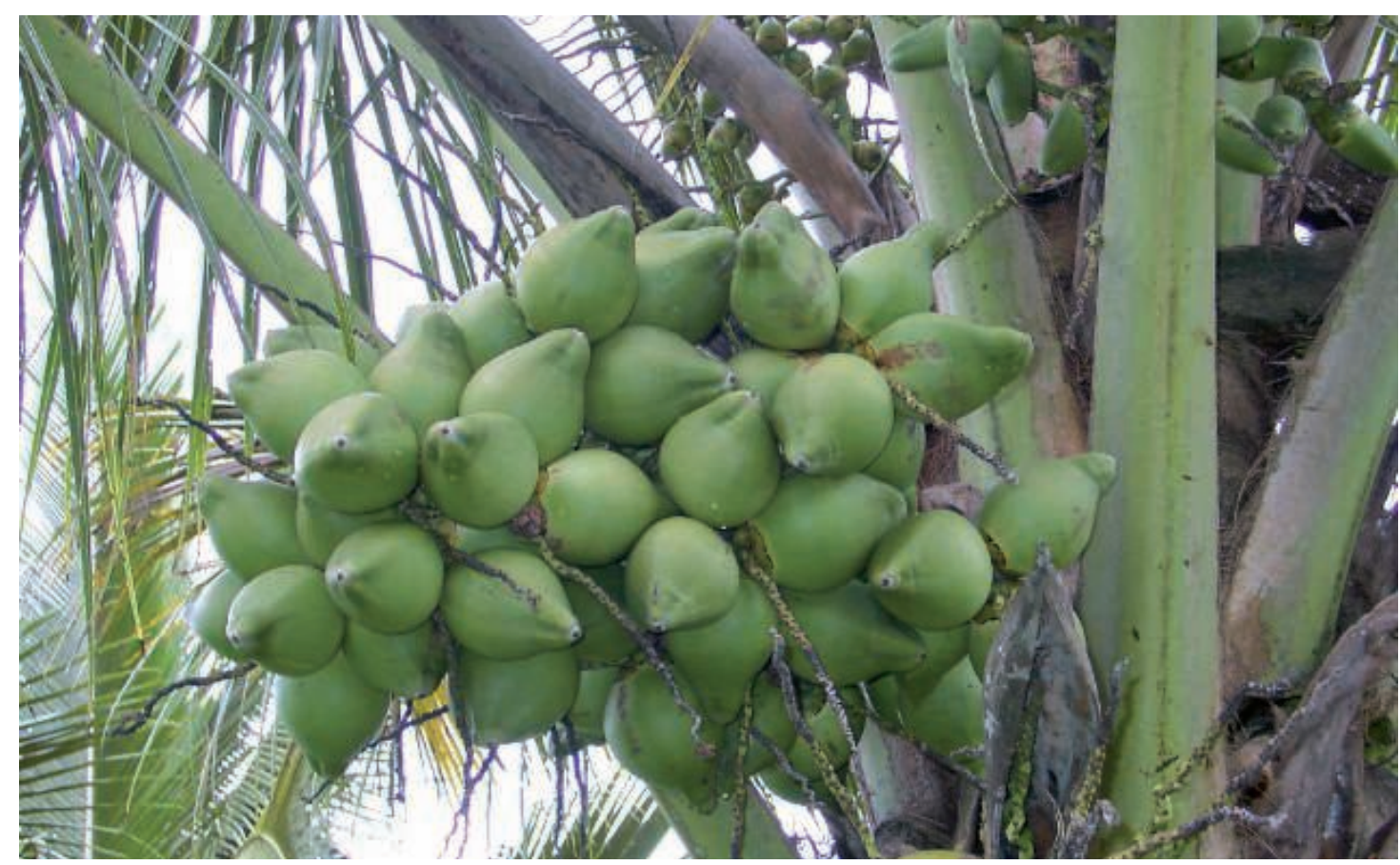

Рното 3. - Catégorie nommée sōgsōg caractérisée par de nombreux petits fruits (CTRAV, Santo, 2003, cliché de Jean-Pierre Labouisse)

2. Les timiat à Vētuboso dénomment l'ensemble des personnes décédées, mais aussi les morts ou autres personnes vivantes malveillantes qui rôdent dans la forêt autour des villages (Hess, 2009).

3. Enquêtes réalisées sur dix sites situés sur dix îles dans le premier cas, et sur quarante sites répartis sur dix-neuf îles dans le deuxième. 


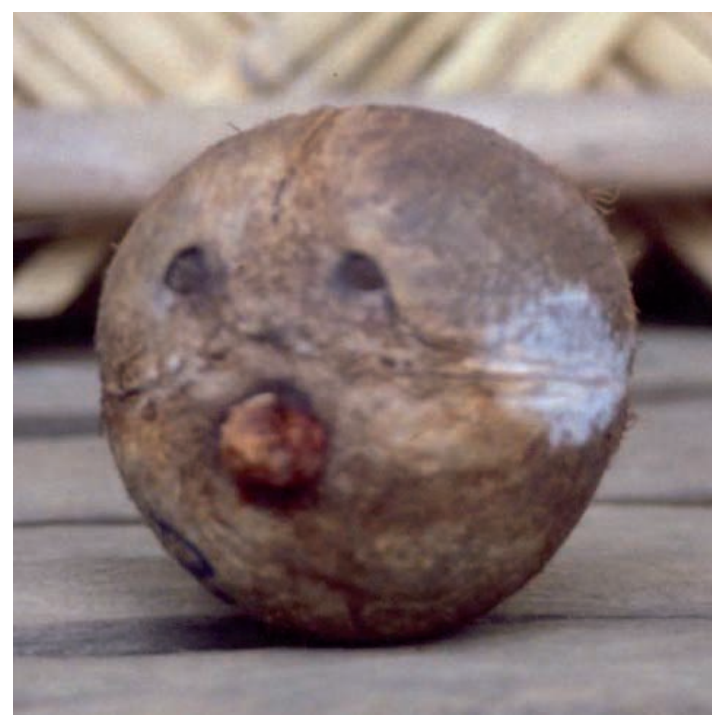

Рното 4. - Catégorie nommée mōtō meteröwö, dont la noix ressemble à une tête de poisson (Vētuboso, Vanua Lava, 2002, cliché de l'auteur)

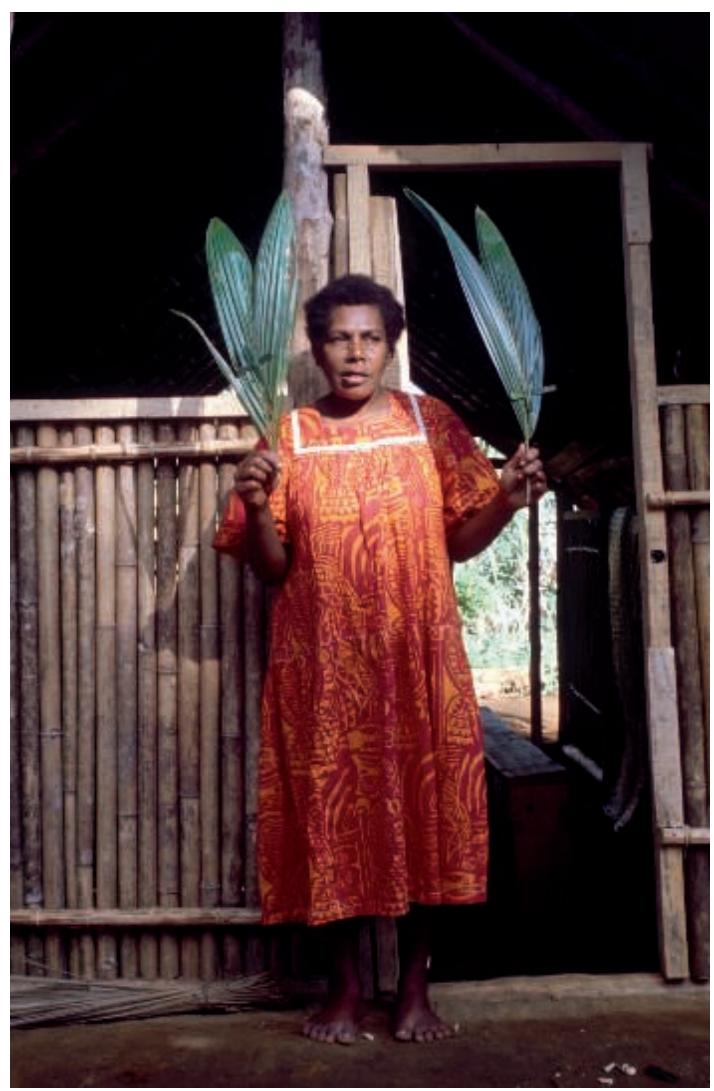

Рното 5. - Femme tenant deux « rameaux » de jeunes pousses de cocotiers décorés de croix, avant de se rendre à l'église pour fêter le dimanche des Rameaux (Vētuboso, Vanua Lava, 2002, cliché de l'auteur) lifiant dans la plupart des cas, explicitement ou par métaphore, une particularité morphologique du cocotier. Trente et un critères d'identification sur $38(82,5 \%)$ concernent l'organe reproducteur, le régime (ou infrutescence) et/ou le fruit.

Les processus de nomenclature et les systèmes de classification sont similaires au Vanuatu (Labouisse et Caillon, 2004) ou même dans le Pacifique. Par exemple, en Nouvelle-Calédonie, nu bange représente une catégorie de cocotiers à très grosses noix dont le mésocarpe est très fibreux (Barrau, 1962). Les Māohi des îles de la Société nomment seize "variétés " différenciées selon des critères morphologiques de l'arbre et des noix (Petard, 1986).

À Vanua Lava, le déterminant en langue vurës est motivé dans 33 cas (87\%), c'est-à-dire qu'il est porteur de sens pour les locuteurs. Il fait référence à une couleur (6 catégories nommées), à la taille (2), à la forme (4) et à la consistance ou au goût (2) ; il peut prendre la forme d'une métaphore qui renvoie à une action (6), au genre humain (2), à un animal (1) ou à une partie de son corps (3), à un végétal (1), à un objet naturel (2) ou domestique (1), à une partie d'un corps humain (1). Il peut encore représenter le nom d'une personne (1) ou d'un héros fondateur (1). On remarque l'absence de références à des toponymes. Quatre déterminants motivés renvoient à un mythe ou une histoire coutumière : mōtō mat est entortillé comme un serpent $\bar{m} a t$, mōtō sialmē est arrivé par la mer ${ }^{4}, \boldsymbol{m o ̄ t} \bar{o}$ qet a été planté par le héros des Banks Qet et mōtō vanvan se promène entre Vanua Lava et les Reef Island 5 .

Cinq déterminants des noms de cocotiers sont immotivés (Dubois et al., 1994) ou non-motiver, c'est-à-dire que les informateurs ne savent pas expliquer la signification du lexème qui n'est pas utilisé dans d'autres registres linguistiques et qui concerne exclusivement les coco-

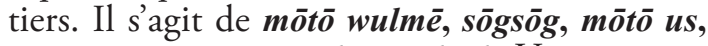
mōtō sörsör et mōtō tak ou taktak. Un terme est immotivé soit parce qu’il est ancien et «appartient au grand fonds " des langues régionales, soit parce qu'il est emprunté à une autre langue (Grenand, 2001-2002 : 222). Les déterminants de ces cinq catégories de cocotiers sont relativement courts traduisant leur usure par le temps et/ou par l'usage (F. Grenand, com. pers.). La catégorie sōgsōg, a même perdu son terme de base. À part le mōtō sörsör, ce sont ces catégories qui sont les plus souvent citées spontanément lorsque les villageois sont invités à énoncer tous les noms qu'ils connaissent. L'immotivation de ces courts déterminants relève vraisemblable-

4. Le $\boldsymbol{m} \overline{\boldsymbol{o}} \boldsymbol{t} \overline{\boldsymbol{o}}$ sialmēe ( cocotier arrivé en flottant ») renvoie aux gros fruits récoltés sur la plage que les planteurs sélectionnent (les petits fruits apportés par la mer ne sont pas plantés) et aux fruits dont la bourre est suffisamment épaisse et l'épiderme imperméable pour les protéger de l'eau salée.

5. Ce groupe de petites îles coralliennes, aussi dénommé Rowa, situé entre Ureparapara et Mota Lava, est un lieu de magie dont sont originaires beaucoup d'histoires coutumières. Elles sont aujourd'hui inhabitées. 
ment de l'ancienneté du nom dans la langue, et non de l'emprunt à une autre langue. On peut également relever que plus une plante est utile ou importante, plus elle sera désignée par un lexème simple opaque (immotivé). Le phénomène d'économie mémorielle explique ce lien afin de pouvoir retenir facilement les noms des plantes les plus couramment utilisées. À l'inverse, les végétaux peu mentionnés portent un nom imagé (lexème productif transparent) que la personne retrouve sans grand effort en regardant la plante (Martin, 1975, en référence au livre de Berlin et al., 1974).

Il existe aussi deux autres catégories de cocotiers issus des programmes de sélection du Centre technique et de Recherche agricole du Vanuatu (CTRAV) situé sur l'île de Santo : l'hybride Nain x Grand du Vanuatu et le cocotier Grand du Vanuatu amélioré (ou GVT Élite) (Labouisse et al., 2004 ; Labouisse et al., 2005). Les cocotiers hybrides, de petite taille et aux noix très nombreuses et rouges, sont facilement identifiables. Cette population d'hybrides est stable en nombre car leur descendance, hétérogène et ne bénéficiant pas de la vigueur hybride, n'est pas replantée suivant les conseils des assistants de l'agriculture. À l'inverse, les agriculteurs récoltent les fruits germés sous les GVT Élite pour les planter dans leurs parcelles. Alors que les hybrides Nain x Grand sont nommés par le terme "hybrides ", les GVT Élite, même s'ils restent identifiés comme tels, peuvent être désignés par les mêmes noms que les cocotiers locaux s'ils présentent une des caractéristiques des 38 catégories locales. Ainsi, les habitants de Vētuboso intègrent du matériel végétal sélectionné par des "hommes blancs " à leur patrimoine local de cocotiers.

Certains cocotiers ont la particularité de " changer " au cours de leur vie. Le déterminant qui les désigne peut alors être complété par le terme, vanvan "se promener", qui renvoie au mōtō vanvan, ce cocotier doué de raison et de mobilité qui ne produit de fruits que pendant six mois ou d'une année sur l'autre. Cette combinaison se rencontre le plus souvent avec le cocotier nommé pour ses nombreuses petites noix, sōgsōg. Le sōgsōg vanvan engendre ainsi au bout de quelques années des noix de taille normale. Ce complément d'information ne fait pas partie du nom courant du cocotier mais est utilisé comme une petite moquerie de son inconstance. Les habitants de Vētuboso s'affranchissent ainsi des changements morphologiques pouvant subvenir au cours de la vie du cocotier ; ce qui importe pour choisir un nom est la particularité première ou l'identité de l'arbre mère.

Â part les noms composés avec le déterminant vanvan, les habitants de Vētuboso ne retiennent pour chaque nom qu'un seul critère pour ordonner la diversité existante. Ils sont ainsi obligés d'en privilégier certains si un cocotier présente

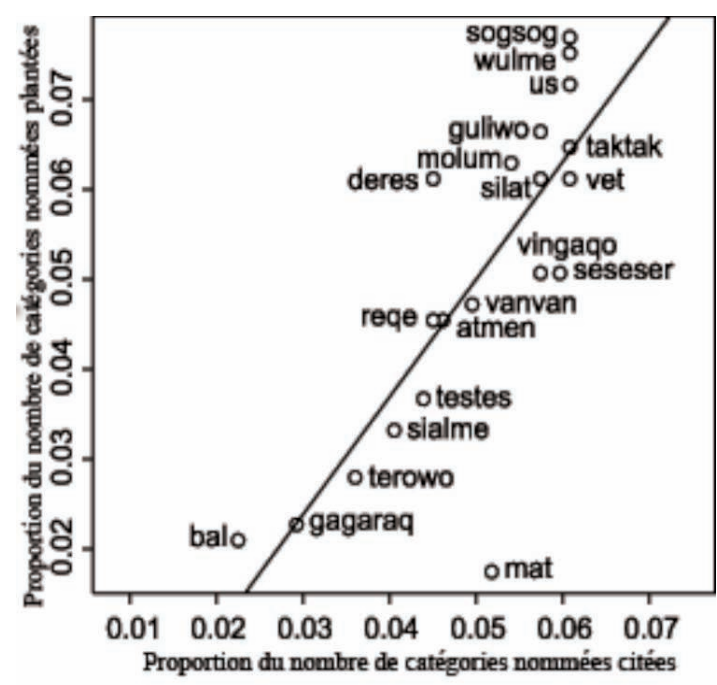

Figure 2. - Proportion de catégories nommées plantées en fonction de la proportion de catégories citées. La corrélation entre ces variables est décrite par une courbe de lissage lowess avec comme paramètre de lissage $\mathrm{f}=0,75$ (Cleveland et al., 1994)

plusieurs caractéristiques discriminantes. Ils choisissent par ordre d'importance la couleur rouge ou rose en anneau autour du pédoncule (mōtō wulmē), puis l'amande sucrée et juteuse (mōtō us), la forme spicata (mōtō taktak), et enfin la taille des fruits (gros fruits du mōtō geluwō ou petits fruits nombreux du sōgsōg). Il est intéressant de noter, qu'à part le mōtō geluwō dont l'intérêt est apparu lors du développement de l'économie de coprah, ces cinq noms sont immotivés. À l'inverse la couleur globale du fruit (rouge, vert, vert foncé et mélangé-kaki) est la caractéristique ayant le moins d'importance : si aucun autre trait n'a pu lui être associé, alors le cocotier portera ce nom général. En conclusion, tous les arbres d'une cocoteraie peuvent être nommés, au moins par leur couleur. Sachant que ce groupe est très peu valorisé par les planteurs, nous avons choisi d'exclure ces quatre catégories nommées $\left(n^{\circ} 1\right.$ à 4$)$ des analyses.

Afin d'identifier une éventuelle structuration des connaissances associées aux catégories nommées de cocotier dans le village, nous avons effectué une analyse des correspondances non symétriques sur les réponses des 56 questionnaires socio-économiques. La connaissance des catégories nommées n'est pas structurée au sein du village. Aucune différenciation par genre, âge, clan ou suivant d'autres critères socioéconomiques (nombre d'enfants, scolarisation, possession de biens, etc.) n’a été mise en évidence.

Les types les plus souvent cités et donc connus sont les cocotiers dénommés sōgsōg, mōtō wulmēe, mōtō us, mōtō tak(tak) et mōtō vet. Les quatre premiers sont les plus anciens d'après leur nom immotivé. Les cocotiers mōtō seseser, mōtō geluwō, mōtō silat et mōtō vingaq $\overline{0}$ sont aussi 
Vingt-trois (61\%) catégories de cocotiers sont associées à un ou plusieurs usages culinaires ou domestiques. Entre parenthèses, est spécifié le pourcentage (>10\%) de personnes enquêtées (56) ayant répondu à un questionnaire leur demandant d'associer une catégorie de cocotiers à un usage.

Usages alimentaires:

- sōgsōg a de nombreuses petites noix recherchées pour leur eau sucrée $(27,5 \%)$ et leur amande gélatineuse sucrée lorsque immature (30,6\%).

- mōtō vet, dont la chair est dure " comme une pierre ", permet l'extraction d'un lait gras $(92,2 \%)$ et offre les meilleurs haustorium des fruits en germination (47,9\%).

- mōtō dēdērēs est réputé pour être le plus sucré de tous, en particulier son eau (31,4\%).

- mōtō us est consommé avec délice au stade mature avec des tubercules car sa chair est molle, juteuse et sucrée $(78,7 \%)$.

- mōtō seseser, dont la bourre fragile peut être détachée facilement de la noix, est apprécié des enfants qui peuvent ainsi facilement ouvrir un fruit pour en consommer la chair mature.

- mōtō tak(tak), les jeunes l'apprécient pour la facilité avec laquelle ils peuvent récolter ses fruits immatures pour en boire l'eau sucrée : en lançant des pierres, les fruits tombent immédiatement des axes centraux dépourvus d'épillets.

Usages non alimentaires:

- mōtō gaan̄añ est conservé dans les jardins pour la beauté de ses fruits et de ses palmes jaunes. Cependant certains se méfient de son aspect maladif.

- mōtō vingaqō était apprécié pour ses longues fibres et mōtō seseser pour ses fibres facilement détachables qui pouvaient ainsi être tressées en cordes imputrescibles servant à assembler et amarrer les pirogues.

- mōtō geluwō a une grande coque utilisée comme récipient, et sa chair abondante est recherchée pour en faire du coprah $(11,8 \%)$.

- mōtō gemetestes a une coque effilée et pointue que les chefs plantaient dans le sol après avoir bu le kava rituel.

- mōtō mölumlum, dont la chair est molle, est recherchée pour faire du coprah car plus facilement décoquable même s'il demande un séchage plus long (52,9\%).

- mōtō vet, à l'inverse, est choisi par d'autres qui préferent faciliter le séchage même si cela suppose de travailler plus durement lors du décoquage $(20,6 \%)$.

- mōtō gagraq, est encore mieux pour le coprah car l'amande se détache tout seul de la coque lorsque le fruit est ouvert à la hache.

- mōtō reqe pousse lentement et produit beaucoup de gros fruits à coprah.

Usages rituels :

- sōgsōg est un cocotier dont les petits fruits immatures sont offerts lors des mariages (76,2 \%).

- mōtō bal est un cocotier à deux stipes. Ces deux stipes ont la forme de mâchoire de cochon. Les fruits de ce cocotier ne pouvaient être utilisés que lors des cérémonies par des chefs coutumiers.

Usages magiques et médicinaux:

- mōtō wulmē a une eau utilisée comme excipient médicinal ou magique, et comme eau de lavage lors de rites coutumiers $(63,8 \%)$.

- mōtō malgias (17\%) et mōtō gōtōtōrōg (8,5\%) ont aussi une eau utilisée comme excipient.

- mōtō gōtōtōōōg qöñqǖñ est spécifiquement utilisé pour soigner les infections urinaires.

- mōtō meteröwö sert comme excipient à des breuvages pour guérir des infections oculaires.

- mōtō lak, le cocotier dont les palmes « dansent» tout le temps, aide les enfants ayant du mal à marcher et des hommes qui cherchent à développer leur capacité à discourir et devenir ainsi un manar, « la personne qui sait ».

ENCADRÉ I - Les usages des catégories de cocotiers de Vētuboso

très connus dans le village de Vētuboso (fig. 2). $\mathrm{Au}$ Vanuatu, les catégories de cocotiers les plus citées (>50\%) sont similaires. Le cocotier albinos, équivalent au mōtō gaan̄an de Vētuboso, celui dont la noix ressemble à une tête de poisson (mōtō meteröwö) et celui dont les fruits sont longs et pointus (mōtō gemetestes) sont également fortement cités au niveau national.

La figure 2 montre aussi une relation entre la connaissance d'une catégorie nommée et sa fréquence de plantation. Aujourd'hui chaque foyer de Vētuboso plante plus de 397 cocotiers. D'après des enquêtes menées in situ dans
24 plantations, 4,5 catégories nommées en moyenne (ou 8,9 arbres nommés) sont plantés à l'hectare, ce qui correspond à une dénomination de 4,8\% des cocotiers plantés. Chaque planteur possède en moyenne plus de 9 catégories nommées, soit 19,7 arbres portant un nom autre que les catégories $1,2,3$ et 4 .

\section{Goûter la diversité à Vanua Lava}

Nous nous sommes intéressés aux qualités organoleptiques de l'eau (saveur) et de l'amande de coco (saveur et texture) sur un échantillon 
limité de catégories nommées. Une évaluation sensorielle au sein d'un jury ( 15 hommes et 15 femmes) en langue vernaculaire a été organisée pour savoir si leurs jugements étaient cohérents avec la nomenclature des cocotiers.

Pour tester la saveur sucrée de l'eau de coco, les échantillons ont été prélevés au même stade de maturité (4 à 6 mois après la nouaison), nommé localement $\boldsymbol{v} \overline{\boldsymbol{o}} \boldsymbol{s}$, qui est connu pour être le stade optimal pour le goût de la boisson. Deux arbres non nommés et deux arbres sōgsōg ont été utilisés pour ces tests. Les eaux de coco jugées comme les plus sucrées provenaient de la catégorie nommée décrite comme telle par les habitants du village (sōgsōg) ${ }^{6}$. Ces eaux de coco contenaient entre 50 et 61 grammes de sucre par litre (degré Brix $\left.^{7}\right)$. Ainsi le classement du jury s'accorde avec la nomenclature locale et les mesures de sucres solubles.

Pour l'amande, les fruits ont été prélevés au stade mereñ (approximativement entre 10 et 11 mois après nouaison), c'est-à-dire le stade optimal pour extraire le lait de coco. L'épreuve triangulaire permet de mettre en évidence de petites différences gustatives entre plusieurs produits comparés deux à deux ${ }^{8}$. Trois échantillons dont deux identiques ont été présentés aux participants qui doivent déceler l'échantillon unique en spécifiant sa qualité distinctive (saveur sucrée, fermeté et sensation " huileuse »). Les participants ne sont pas capables de différencier des amandes provenant de différents fruits récoltés sur un même régime. Ceci vient confirmer les résultats de Mialet-Serra et Thaler (2003) selon lesquels tous les fruits d'un même régime sont au même stade physiologique et possèdent des caractéristiques physicochimiques équivalentes.

En goûtant des amandes issues des quatre catégories nommées distinctes (mōtō vet, mōtōo us, mōtō dēdērēs et mōtō malgias), les dégustateurs se sont révélés capables de les différencier suivant leur saveur sucrée ; le cocotier nommé pour sa chair molle, juteuse et sucrée est perçu comme le plus sucré (mōtō us). En revanche, le jury n'a pas différencié ces mêmes échantillons selon leur fermeté et la sensation " huileuse " en bouche.

Ces tests montrent, qu'au moins pour la saveur sucrée de l'eau et de l'amande, les habitants de Vētuboso s'accordent entre eux et que leur jugement est cohérent avec la nomenclature locale des cocotiers : le sōgsōg n'a pas volé sa réputation de porter des fruits à eau et amande sucrées, et le mōtōo us est bien le cocotier à chair molle et sucrée.

Ainsi, classification et nomenclature du cocotier répondent à des critères tant morphologiques, floristiques, écologiques, physiologiques, que fonctionnalistes ou symboliques. Ce système de classification renvoie au système de représentation, soit au "système rendant compte de la façon dont est organisé, au sein d'une culture particulière, l'ensemble du monde naturel » (Friedberg, 1968).

\section{Usage ordinaire du cocotier}

Le cocotier est très souvent surnommé « arbre de vie " (Ohler, 1984) pour la multiplicité de ses usages tant alimentaires que domestiques et son rôle dans l'économie familiale. Les usages spécifiques des cocotiers nommés de Vētuboso, sont mentionnés dans l'encadré 1 . Depuis que le Vanuatu a intégré une nouvelle économie fondée sur le marché, que sont devenus ces usages ?

\section{Fruit, légume et condiment}

La noix de coco est à la fois un fruit, un légume (au sens alimentaire et non botanique du terme) et un condiment. Elle est consommée comme fruit à un stade immature (vōs) pour sa chair gélatineuse et son eau, toutes deux savoureuses et sucrées ${ }^{9}$, et à un stade avancé de germination, sol, pour consommer l'haustorium, un tissu qui se nourrit des réserves de l'albumen dans les fruits germés. Consommée comme fruit, la noix de coco est classée parmi la " nourriture légère " wëmën̈ël comme la canne à sucre (töv, Saccharum officinarum L.). Les fruits germés ouverts constituent aussi la base de l'alimentation des cochons

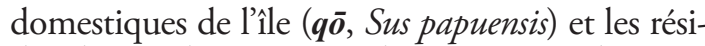
dus d'amande râpée sont donnés aux poules $(\boldsymbol{t} \overline{\boldsymbol{o}})$.

En tant que légume, le cocotier appartient à la classe des accompagnements nommée bigbig. L'unique bourgeon terminal du cocotier est un mets d'autant plus recherché que pour le consommer il faut sacrifier l'arbre entier. À Vētuboso même si les villageois connaissent cet usage, ils ne le mettent pas en pratique car cela impliquerait la mort du cocotier. L'huile recueillie dans la cavité des noix germées est utilisée pour graisser les feuilles d'héliconia (damat, Heliconia indica Lam. ou dēnin, Cominsia gigantea $\mathrm{K}$. Schum.), afin qu'elles n'attachent pas à la

6. Pour analyser les données, un $\chi^{2}$ modifié de Friedman et le plus petit écart ont été appliqués.

7. Soit la teneur en sucres solubles exprimée en grammes par litre d'eau de coco mesurée à l'aide d'un réfractomètre manuel à compensation de température.

8. Pour analyser les données, on utilise la méthode de Sauvageot et Dacremont (1995).

9. Lorsqu'un fruit d'une infrutescence est dépourvu d'eau, les habitants de Vētuboso disent qu'un serpent lui est passé dessus, matgosogolo (mat, serpent ; sogolo, dessus). 


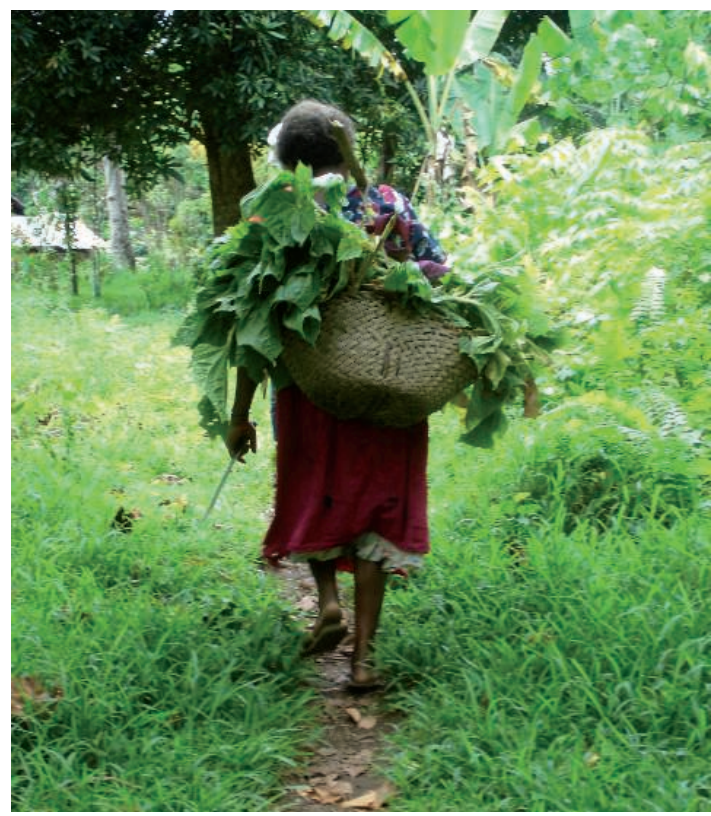

Рното 6. - Femme de la côte est de Vanua Lava transportant du choux canaque dans un panier tressé avec des folioles de cocotier (Qanlap, Vanua Lava, 2010, cliché de l'auteur).

pâte de tubercules ou de fruits lors de la cuisson du laplap, le plat national du Vanuatu. Les habitants de Vētuboso la consomment aussi telle quelle avec un morceau de taro cuit (qiat, Colocasia esculenta (L.) Schott).

Toujours parmi les accompagnements bigbig, le lait de coco est préparé chaque jour à partir de noix de coco mature, merē̄. L'amande était autrefois râpée avec un coquillage de mer, wertur, et aujourd'hui avec une spatule en fer dentée et fixée à une planche sur laquelle on s'assoit. L'amande râpée, additionnée ou non d'eau selon les recettes, est malaxée et pressée pour en extraire un lait riche en matières grasses et en arômes. Ce lait est éventuellement filtré à travers les tissus fibreux que l'on trouve à la base des palmes de cocotier, denen. Le lait de coco peut être consommé frais ou cuit ; il accompagne toujours d'autres aliments.

Le lait de coco mélangé à l'eau de cuisson permet de cuire des feuilles, principalement celles du chou canaque (sasar, Abelmoschus manihot (L.) Medik), qui accompagneront les cormes de taro.

Le lait de coco peut être aussi mélangé à l'eau de cuisson ou versé pur dans les marmites où sont cuits des tubercules et des fruits coupés en morceaux (salōqōs) ou râpés et mis en boulettes (löt gëvtun). Si le lait n'est pas cuit, les tubercules et les fruits ainsi préparés seront dénommés wōrqarqar. Enfin, le plat consistant à mélanger ces féculents avec de la chair de coco râpée porte le nom de bigtöw.
À Vanua Lava, le nalot ou löt, dont les habitants des îles Banks passent pour être les spécialistes, est une pâte préparée principalement avec des taros entiers cuits puis écrasés au pilon. Les löt portent des noms différents selon la nature et la préparation de la garniture. Si les löt aux nangailles et aux velles sont les plus valorisés au village, les plus communs restent ceux au lait de coco car les noix sont disponibles tout au long de l'année. Le löt est recouvert de lait de coco frais (wirmamēgin $)^{10}$ ou cuit légèrement pour former une crème épaisse, soit une concentration par chauffage du lait de coco. Le plat est nommé löt wirsal si le lait de coco est cuit dans des coques évidées dans lesquelles sont introduites des pierres chaudes, löt teqeseg s'il est cuit enveloppé dans des feuilles d'héliconia posées sur des braises, et löt wötöñ̈̈ s'il est cuit par des pierres chaudes avec des feuilles aromatiques dëm̄ël dans des coques. Le lait peut être cuit plus longuement jusqu'à ce qu'il forme des particules rougeâtres très sucrées : le lait de coco du löt salsim est cuit dans des coques sur des pierres chaudes et celui du löt vasgêr est cuit dans des coques sur des braises.

Pour préparer un laplap, les cormes ou fruits (de taro, manioc (maniok, Manihot esculenta Crantz), ignames (dëm, Dioscorea alata ; tamag, D. esculenta; $\boldsymbol{q} \overline{\boldsymbol{o}} \overline{\boldsymbol{\sigma}} \boldsymbol{r}, \mathrm{D}$. nummularia), bananes (vetel, Musa spp.) sont râpés crus, puis empaquetés dans des feuilles d'héliconia et cuits dans un four à pierres. Quatre types de laplap sur sept, sont préparés avec du lait de coco. Celui-ci peutêtre versé frais (wir, pour le nom de la préparation), mélangé encore frais à la pâte de tubercules pour être cuits ensemble (wōrkelkel), inséré sous forme de crème blanche dans des trous de la pâte avant cuisson (wōrōsala) au four à pierres ou encore servir d'eau de cuisson à des boulettes de tubercules râpés (wewe).

Que ce soit pour nommer un löt ou un laplap, on note que c'est la manière de préparer le lait de coco (ou autres noix) qui déterminent la nomenclature et non le mode de cuisson des taros ou le type de tubercules ou de fruits utilisés. Le lait de coco est le critère déterminant dans la classification des plats alimentaires du village.

Dans le village de Vētuboso, comme partout en zone rurale au Vanuatu, il a été estimé qu'en moyenne six noix de coco sont consommées chaque jour par les membres d'un foyer et trois par les animaux, principalement les cochons domestiques. Or un ancien de Vētuboso considère justement que les hommes se comportent aujourd'hui comme des " cochons » en consommant quotidiennement des noix de coco. Autrefois, la cuisson et les préparations culinaires au lait de coco étaient exceptionnelles; chaque famille

10. Il est préparé pour « assurer une bonne croissance des enfants » car il est moins gras que les autres selon Eli Field Malau. 
ne possédant qu'environ dix arbres, l'apport en huile végétale était principalement complété par les nangailliers et les velliers. On peut justement noter quaucune catégorie nommée n'est associée à un usage de lait de coco (encadré 1). Le régime alimentaire des villageois s'est certainement modifié avec l'augmentation du nombre de cocotiers et l'abondance de leurs fruits.

\section{L'arbre aux mille usages domestiques}

À chaque usage correspond une partie du cocotier : feuille, nervure, coque et bourre. Les palmes entières sont posées sur les toits en feuilles de sagoutier (doot, Metroxylon warburgii Becc.) pour les protéger des vents violents. Si une partie du toit est emportée lors d'un cyclone, des palmes rapidement et sommairement tressées boucheront les interstices pour éviter que le vent, en s'engouffrant, n'emporte le reste du toit. Tressées en nattes serrées, $\overline{\boldsymbol{m}}$ isiak, les palmes peuvent servir de toits pour les maisons éphémères dans les jardins. Les folioles souples situées à la pointe de la palme sont tressées en éventail, dêrivriv. Les plus solides sont tressées en de nombreux paniers ayant chacun un usage bien particulier (encadré 2). Elles sont aussi séchées à l'intérieur des maisons pour allumer le feu du matin ou pour éclairer son chemin la nuit. Les nervures des folioles sont assemblées en balai, et chacune d'entre elles est utilisée pour vérifier la cuisson d'un tubercule en train de cuire dans une marmite (metesusus).

Le stipe de cocotier est utilisé comme pilier dans la construction des maisons. Brûlant lentement, il est également un bon combustible ${ }^{11}$ comme les coques vides; ces dernières donnent à la nourriture, poisson ou igname nummularia, un goût et une texture particuliers. Elles servent également de récipients, en particulier celles qui sont allongées et pointues car elles peuvent ainsi être plantées dans la terre par le buveur de kava (gēe, Piper methysticum G. Forst). Avant l'utilisation de grattoirs industriels, les coques permettaient de râper l'épiderme des cormes de taro, et servent encore à creuser le four à pierre.

Le tissu fibreux à la base des palmes et la bourre de coco servent de passoire (sēn) pour filtrer les débris de racine de kava ou les débris d'amande du lait de coco. On peut s'essuyer les mains avec

Les paniers en cocotier de Vanua Lava sont confectionnés avec les nervures et les folioles dénervurées ou non. Les femmes choisissent la palme de cocotier pour son aspect (couleur verte, ni trop jeune car moins résistante, ni trop vieille car abîmée et cassante) et pour sa taille selon la dimension recherchée du panier. Les folioles à la base de la palme sont préférentiellement utilisées. Neuf types de paniers de cocotier ont ainsi pu être recensés sur Vanua Lava, principalement dans le village de Vētuboso ${ }^{12}$.

1. Le metedērēs, panier tabu ou prohibés pour les hommes reclus dans la maison des hommes (gemel), est confectionné avec dix folioles dénervurées mais attachées entre elles par une partie du rachis de la palme. Il n'est plus utilisé aujourd'hui.

2. Le toq, généralement composé avec des feuilles de pandanus plus robustes, peut également être tressé avec des folioles de cocotier doublées pour le rendre plus solide. Il est utilisé pour stocker et sécher des nangailles dans leur coque au-dessus du feu. Une fois sèches, elles sont conservées dans un panier exclusivement en pandanus, le toq venit. Par contre, les nangailles sèchées sont vendues dans des toq en cocotier.

3. Le wōsusrō existe en version " cocotier ", plus large et plat, et en version "pandanus " pour enfermer les restes alimentaires et les déjections des enfants afin de les protéger de la mauvaise magie. Le wōsusrō en cocotier était utilisé pour porter le bois de chauffage et la nourriture au cours des fêtes. Il n'est plus d'usage aujourd'hui.

4. Le matqēt est une sorte de plateau avec deux anses sur lequel on dispose de la nourriture cuite comme le laplap. Après avoir servi, il est accroché au mur par une anse.

5. Le meterōwa kulē̄o ("dos de tortue ») ressemble au précèdent mais il est tressé avec des folioles non dénervurées. On l'utilise pour placer de la nourriture cuite.

6. Le $\boldsymbol{q} \overline{\boldsymbol{o}} \boldsymbol{t} \boldsymbol{u} \overline{\boldsymbol{o}} \boldsymbol{w}$ vir, tressé avec des folioles, permet de conserver dans la cuisine les coquillages utilisés pour râper la chair de coco.

7. Le bor, un panier qui peut être fermé, est le plus commun. Il permet de ramener les tubercules du jardin et de les transporter vers le marché ou vers d'autres villages ou îles. Si un invité ne se rend pas chez ses hôtes, ces derniers accrochent sur leur porte ce panier non achevé afin de se moquer de lui.

8. Le wagateteg doté d'une anse en écorce d'hibiscus (vër, Hibiscus tiliaceus L.) est utilisé pour porter le bois de chauffage au jeune couple lors de leur cérémonie de mariage.

9. Le grand wēgēt permet de ramener de la nourriture non cuite du jardin, alors que le petit $\boldsymbol{w} \overline{\boldsymbol{e}} \boldsymbol{g} \overline{\boldsymbol{e}} \boldsymbol{t}$ permet le transport de nourriture non cuite depuis la plage. Très commun, il se conserve longtemps au-dessus du feu.

ENCADRÉ 2 - Les paniers en cocotier de Vanua Lava

11. Cependant, nous n'avons jamais eu l'occasion en deux ans d'observer ces deux derniers usages du stipe.

12. Ces informations ont été récoltées grâce au travail de Kate Ruth, habitante de Vētuboso. 
la bourre, l'utiliser comme papier hygiénique $(\boldsymbol{m e n})$ et comme combustible lorsqu'elle a été séchée sous abri.

Les nervures des palmes ou la bourre de coco étaient jadis tressées en corde ; la corde en bourre imputrescible, était particulièrement prisée pour l'amarrage et l'assemblage des éléments des pirogues et sert encore aujourd'hui à ligaturer le bout pointu des flèches et des lances. Aujourd'hui les cordes synthétiques commerciales sont omniprésentes ; ces dernières sont non seulement responsables de l'érosion d'un savoir-faire mais aussi de la désélection d'une catégorie de cocotier à fruits longs et à bourre épaisse. Ainsi à Vētuboso, l'utilisation de cordes synthétiques a entraîné, en une seule génération, la disparition du mōotō vingaq̄ō. Cette catégorie au nom familier, dont l'usage est connu de tous, ne se rencontre pratiquement plus que chez les arbres de plus de 60 ans. Cette richesse en bourre utile pour la confection des cordages est un facteur défavorable pour un planteur produisant du coprah car avant de fendre la noix pour en extraire l'amande, le fruit doit être débourré.

\section{Un rôle économique irremplaçable}

"Le cocotier, c'est de l'argent » est une réponse fréquente que l'on obtient à la question « à quoi sert le cocotier? ». Par exemple, les noix de coco peuvent être directement utilisées comme monnaie. Ainsi, en échange de gâteaux, une femme du village accepte d'être payée par des noix de coco suivant le tarif : 10 noix pour 20 vatus, soit 15 centimes d'euro. Mais c'est lorsqu'il est transformé en coprah que le fruit du cocotier assure la majorité des revenus monétaires du village à la hauteur de 210 euros par foyer et par an (Caillon, 2007). Bien que le travail de production du coprah demeure difficile, les habitants de Vētuboso n'ont pas encore la possibilité de développer d'autres activités économiques car les ressources marines sont pauvres ${ }^{13}$, et l'accès au marché de Sola, la capitale administrative de la Province de Torba située à trois heures de marche, est difficile. Les voies de communication maritime permettant la liaison avec d'autres îles sont aussi difficiles. Les bateaux de marchandises collectant le coprah s'ancrent dans la baie de Vureas, à 20 minutes en contrebas du village avec 150 mètres de dénivelé. Leur fréquence est irrégulière et imprévisible : entre un et dix par semestre. Mais ces réseaux de communication se- raient encore plus désorganisés si l'économie du coprah venait à disparaître : la vente de coprah permet de désenclaver les régions isolées du Vanuatu et de maintenir les services publics comme les écoles et les dispensaires (Labouisse, 2004).

La communauté de Vētuboso fonctionne sur le système du big men; l'importance politique et sociale s'accroît avec la richesse personnelle. Traditionnellement, plus un homme avait de cochons à sacrifier, cochons souvent nourris avec des noix de coco, plus il avait de taros à cuire pour nourrir les autres membres de la communauté lors d'une grande fête, et plus son statut social était valorisé. Si l'important est d'être riche, au lieu de cultiver des taros et d'utiliser le système de don et contre-don pour accumuler des richesses, un homme peut exposer sa richesse en achetant de la viande et du riz avec de l'argent. À Vētuboso, les principaux moyens d'obtenir cet argent résident dans la vente de coprah, la marge réalisée dans de petites échoppes sur le commerce de produits importés ${ }^{14}$, la vente de bétail, et celle de kava dans des bars du village ou plus rarement à Sola. Pour un homme du village, la vente du coprah est donc un moyen de capitaliser et de monter en grade dans la société ${ }^{15}$ ou même de démontrer son aptitude à devenir père de famille. Planter une cocoteraie pour un jeune est un moyen de marquer son territoire dans le village. Il démontre ainsi sa capacité à anticiper les besoins monétaires de sa future famille (un cocotier ne fructifie souvent que sept ans après plantation). Construire une maison, cultiver des taros et planter une cocoteraie détermine la valeur d'un mari et d'un père de famille.

\section{Le cocotier, un objet symbolique}

\section{Des usages festifs et cérémoniels}

Le cocotier, comme contenant, nourriture ou objet offert, a toujours sa place dans toutes les fêtes et les cérémonies qui scandent la vie d'un homme ou d'une femme du village. Ces événements ont déjà été détaillés dans un précédent article du Journal de la Société des Océanistes (Caillon and Lanouguère-Bruneau, 2005) ; j'insisterai ici sur les rites anciens et actuels impliquant le cocotier.

Lorsque les hommes participaient à des institutions statutaires comme la hiérarchie du soq,

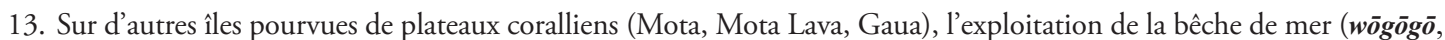

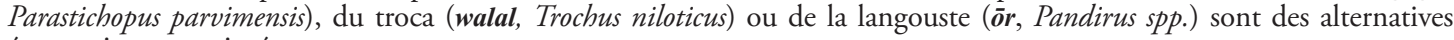
économiquement intéressantes.

14. Principalement du riz, des boîtes de bœuf et de poisson en conserve, du savon, du kérosène, des allumettes et quelques vêtements.

15. Sur Mota Lava, V. Lanouguère-Bruneau (2002) remarque que, à l'inverse d'un travail en dehors de l'île (en ville ou sur des plantations) ou la propriété d'une petite échoppe, les cocotiers ne semblent pas être une source de prestige sauf s'ils permettent d'aider quelqu'un. 
ils pouvaient y acquérir des masques timiat principalement confectionnés avec de la bourre de coco. Aujourd'hui, ces masques ne sont plus confectionnés dans le village.

Dans les temps anciens, la sage-femme donnait de l'eau de coco et du löt au nouveau-né pour vérifier qu'il avait un trou pour l'eau et un autre pour les aliments solides (Speiser, 1996 [1923] : 256). Si l'accouchement était douloureux pour la jeune mère, un spécialiste local de la médecine "coupe la jeune pousse d'un très jeune cocotier, profere une incantation sur la noix dont le lait est donné à la mère pour qu'elle le boive ; la noix vide est ensuite enterrée sous le seuil avec l'ouverture en haut » (Speiser, 1996 [1923] : 255). De plus, le cinquième jour de la naissance, s'il s'agissait d'un garçon, le clan ou $\boldsymbol{v} \overline{\boldsymbol{e}} \boldsymbol{n} \overline{\boldsymbol{e}} \overline{\boldsymbol{m}}$ du père affrontait celui de la mère en lançant des noix de coco. Ces pratiques ne sont plus en usage aujourd'hui.

Le mariage suit traditionnellement un long processus au cours duquel la famille du garçon doit " réserver " puis " payer " la femme désirée. Dans les temps anciens, lorsque la jeune fille était trop jeune pour être mariée, la famille de son futur époux la réservait au cours de la cé-

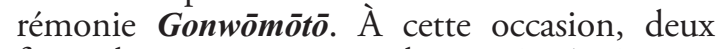
fruits de cocotier étaient plantés côte à côte, attachés ensemble par leur bourre pour symboliser la solidité du contrat entre les deux familles. Les fruits attachés doivent être en germination si l'homme est plus âgé que la femme. La mort de l'un des deux cocotiers avant que l'union finale n'eût été établie, était interprétée comme le passage à la mort de l'un des deux partenaires. Il ne faut pas confondre cette cérémonie avec

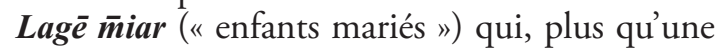
cérémonie, marquait toute la période pendant laquelle l'enfant-fille vivait avec sa belle-mère et l'enfant-garçon était élevé par son père dans la maison des hommes, jusqu'à ce qu'ils atteignent l'âge de se marier, la puberté ${ }^{16}$. Pour conclure une telle alliance, des fruits germés de cocotier et autres arbres à planter, ainsi que de l'argent, étaient offerts à la famille de la petite fille. Si ces deux premières cérémonies ne sont plus pratiquées, celle dénommée Qalēgor l'est toujours. En bichlamar, les Ni-Vanuatu utilisent le verbe blokem pour bloquer ou réserver. Lorsque la famille du futur marié n'a pas suffisamment d'argent pour payer l'ensemble de la somme due, la jeune fille est réservée par l'apport d'une somme d'argent. La présence de fruits de coco germés est indispensable lors de cette cérémonie. Normalement, le futur couple ne peut vivre ensemble que lorsque la dernière cérémonie, Dala ge est achevée, c'est-à-dire lorsque le jeune homme a réuni la somme de 40000 vatus, soit 310 euros.

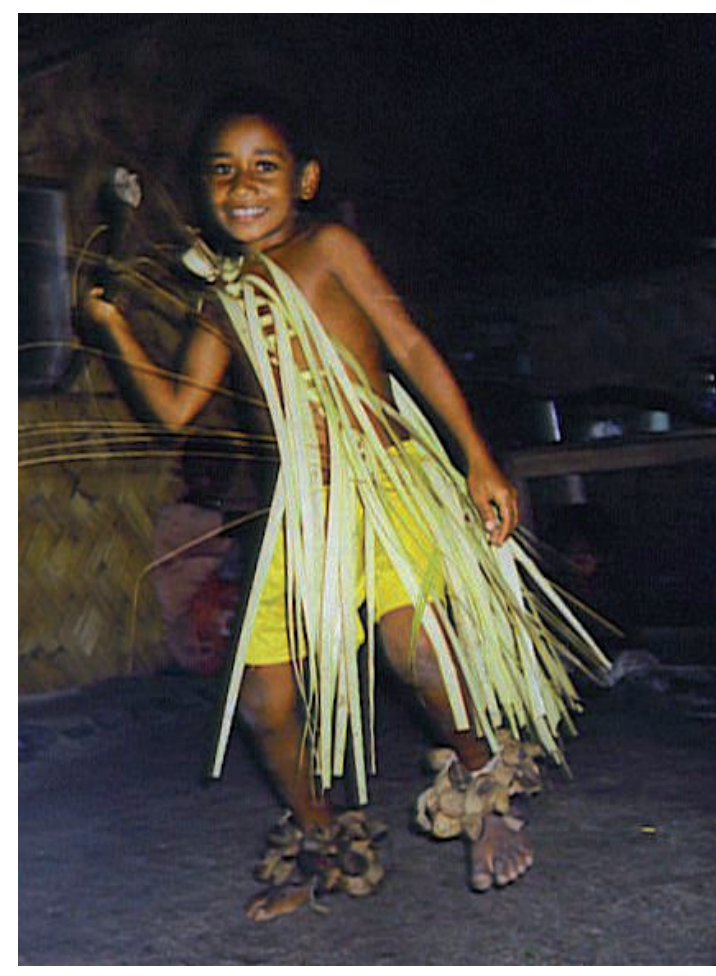

Рното 7. - Jeune garçon dansant avec une tunique à base de folioles de cocotier (Lesereplag, Ureparapara, 2003, cliché de l'auteur)

À cette occasion, des plantes sont offertes pour être cultivées incluant des noix de coco germées, ou être consommées par les personnes déjà mariées comme les noix de coco immatures. On offre également des objets domestiques comme des paniers tressés avec des folioles ou des nervures de cocotier ou des nattes. Pour certains habitants de Mota Lava, le fait de payer une femme avec des fruits germés constitue une reconnaissance de la capacité reproductrice de la femme, alors que les cadeaux de noix de coco vertes sont, comme le sous-entend le mythe de la danse Natmatmumgēl, le signe d'une "dynamisation » de la vie nouvelle des jeunes mariés (LanouguèreBruneau, comm. pers.).

Les cérémonies suite à un décès sont encore aujourd'hui marquées de plusieurs étapes comme la mise en terre, les repas communautaires, la cérémonie Tuleg ununseg pour le partage des terres, la distribution du collier d'hibiscus, $\boldsymbol{g} \boldsymbol{a}$ nar, qui instaure les tabous alimentaires et qui, un an plus tard est brûlé dans de la bourre de coco. Avant l'aube du cinquième jour, les biens du défunt étaient auparavant détruits lors de la cérémonie Vono $\overline{\mathbf{m}} \overline{\boldsymbol{l}} \mathbf{l} \overline{\boldsymbol{o}}$. Plus le mort était respecté, et plus la personne chargée de détruire les biens, en particulier les cocotiers, était enthousiaste dans sa tâche. Cette personne était payée par les enfants en argent et en taros. Bien que présente 
dans le discours local, cette dernière cérémonie n’a plus cours. Les cocotiers ne sont plus détruits en raison de leur nombre, de leur rôle patrimonial et d'épargne familiale. Autrefois, la destruction des arbres permettait de libérer la terre. La logique de capitalisation qui s'installe petit à petit au Vanuatu, attribue au cocotier un nouveau pouvoir, celui de capturer le foncier sur plusieurs générations dans un pays où, à l'inverse de ce qui est planté, la terre n'appartient pas traditionnellement aux individus.

\section{Des usages médicinaux et magiques}

Le cocotier, par les vertus de sa bourre, de son eau ou de son écorce, est un arbre médicinal. Il permet de soigner des infections dermatologiques, urinaires et généralisées. L'huile naturelle de fruit germé (sous l'haustorium) permet de soigner des blessures à la tête et d'éloigner les mouches des blessures. Lorsque l'on est blessé par les fleurs de velliers qui provoquent des excroissances sur la peau, l'eau stagnant dans des spathes de cocotier au sol est frottée sur les blessures. Pour soigner la cigua (la ciguatera ou gratte $)^{17}$, la chair d'une noix pas encore sèche prise au stade gēbibiag, doit être râpée avec le coquillage wertur pour en extraire le lait, qui filtré avec le tissu fibreux denir, sera bu par le malade malgré son mauvais goût d'huile. Les infections urinaires sont soignées par la substance extraite de l'écorce du stipe d'un cocotier aux fruits verts foncés dénommé mōtō gototorog qon̄qōn. Prélevée sur le côté du stipe orienté est, la seconde peau plus blanche et plus souple du stipe est broyée puis filtrée avec les tissus fibreux à la base des palmes. Il faut boire l'ensemble de la solution $^{18}$. Pour stopper une infection généralisée dont le symptôme est l'émergence de ganglions douloureux à l'aine (wotan), une corde de fibres de coco doit être nouée autour du pouce et du petit doigt du pied de la jambe souffrante. Un habitant de Vētuboso se sentant faible et fébrile a été guéri par un homme de Gaua qui a placé un fruit de coco vert foncé à la tête de son lit. Au matin, le fruit a été ouvert sur une feuille de taro

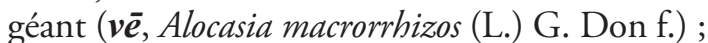
l'eau était trouble comme si elle contenait des débris de taro que quelqu'un d'autre essayait de lui voler pour l'affaiblir.

Il a fallu de nombreux mois pour recueillir les usages médicinaux du cocotier. Les villageois, pourtant déjà interrogés sur le sujet, ne parlent que lorsqu'ils sont confrontés à la maladie. Il ne s'agit pas semble-t-il d'une réticence à divulguer des secrets médicinaux sachant que nous avons facilement pu en collecter pour d'autres plantes locales. L'explication serait que les villageois n'associent plus le cocotier à des propriétés médicinales.

Contrairement à leur utilisation médicinale, les usages magiques du cocotier sont secrets. L'ensemble des "recettes " décrites ci-dessous sont incomplètes et n'ont jamais été divulguées aux autres membres de la communauté. Qōto$\boldsymbol{n o ̈ g} \overline{\boldsymbol{o}} \boldsymbol{s} \overline{\boldsymbol{o}} \boldsymbol{w}$, la noix sans sa bourre, sèche et évidée mais non brisée, est utilisée comme diffuseur de feuilles magiques, par exemple enfouie à l'entrée des bassins de taros pour éliminer les ravageurs. Le cocotier magique par excellence est celui qui, immature, a un anneau rouge autour de l'attache du pédoncule au-dessus des pores de germination : mōtō wulmē. L'eau de cette catégorie de cocotier est en général utilisée comme excipient pour un grand nombre de recettes magiques dans lesquelles entrent d'autres plantes. Du temps de la hiérarchie du soq, l'homme qui passait son premier grade ne pouvait pas, entre autre, se laver pendant cent jours alors qu'il était enfermé dans la maison des hommes. À sa sortie, il devait se laver avec un seul fruit du stade mël (contient de l'eau mais pas encore d'amande) d'un cocotier à anneau rouge. Ce cocotier a également une utilisation magique pour faire venir les poissons nommés röwö. Dans une noix du stade mël bouchée par des galets de la plage, des feuilles magiques sont enfermées. Lorsque la marée est haute, cette noix doit être discrètement et invisiblement enterrée à la limite de la ligne d'eau. Cinq jours plus tard, on peut observer l'arrivée des poissons par l'obscurcissement de la couleur de la mer. Pour les stabiliser près du rivage, des algues consommables sont multipliées sous l'action d'une autre feuille jetée dans la mer.

Si un enfant ne sait pas bien marcher, la mère le présente à un cocotier dont les folioles bougent tout le temps même lorsque le vent s'arrête, le cocotier mōtō lak. Le père agite de haut en bas des folioles de cocotier devant puis derrière l'enfant. Si quelqu'un a besoin d'avoir un discours éloquent, de "s'ouvrir le gosier » en bichlamar, il doit boire l'extrait, filtré dans les tissus fibreux du cocotier, de huit folioles de ce même arbre écrasées à la pierre et mélangées à de l'eau de mer. Avec les débris végétaux, le corps doit être frotté deux fois devant et deux fois derrière. Puis, ces

17. Après ingestion de poissons herbivores et de leurs prédateurs, la cigua se traduit par l'accumulation dans les muscles et les viscères de substances naturelles toxiques provenant d'une algue unicellulaire qui vit accrochée aux substrats macroalgaux colonisant les coraux morts.

18. À Hokua, nord Santo, un liquide rougeâtre est extrait de l'écorce du cocotier pour les femmes qui viennent d'accoucher. La bourre de coco, en décoction, est également utilisée à Pentecôte (Atartabanga) pour soigner les infections du tube digestif (diarrhée). On peut également noter qu'aux Torres, après que les arbres d'un futur jardin aient été brûlés, des feuilles de cocotiers sèches en feu sont traînées sur le sol pour tuer par la chaleur les scarabées ravageurs Papuana spp. (C. Mondragón, com. pers.). 
débris sont jetés dans la quatrième vague pour que le corps puisse prendre la force de celle-ci. Outre la capacité à discourir, on acquiert ainsi la possibilité d'être un manar, "la personne qui sait " les danses coutumières et les rites, mais aussi l'ensemble de la flore et de la faune.

En mars 2002, un couple de Vētuboso a fait appel à un "sorcier " de Santo car les deux se plaignaient de maladies chroniques. Le sorcier trouva deux récipients en bourre de coco contenant de la craie, un crâne miniature sculpté, des débris d'os humain et de la cendre ; cette " recette » aurait été empruntée à des sorciers de Pentecôte ${ }^{19}$. L'un était enfoui dans la terre, sous le lit de leur maison sur pilotis. Le deuxième était enterré sous un cocotier que le propriétaire malade avait planté. Pour expliquer ce geste, des habitants du village nous expliquèrent que le cocotier symbolise l'homme avec ses fruits en forme de visage : les deux pores non germinatifs représentent les yeux et le pore germinatif la bouche à travers de laquelle on peut s'abreuver. Si le « sorcier " n'avait pas trouvé le paquet magique en bourre de coco, alors la mort de l'arbre aurait précédé celle de l'homme qui l'avait plantée ${ }^{20}$.

\section{Le cocotier, un arbre anthropomorphique}

Le cocotier " procure de la nourriture à consommer, et des métaphores pour vivre " (Giambelli, 1998 : 153). Ces métaphores s'inspirent principalement de la forme humaine du cocotier. Certains traits biologiques, morphologiques et comportementaux du cocotier peuvent être associés à ceux des humains ${ }^{21}$. Dans le village de Vētuboso, l'anthropomorphisation du cocotier est identifiable à plusieurs niveaux : le mythe d'origine, la ressemblance de la noix de coco à un visage ou à un sein féminin, la liaison mortelle entre le cocotier et l'homme, sa présentation comme un être rusé et le développement biologique du cocotier proche de celui d'une femme.

Tout a commencé lorsque le cocotier poussa de la tête d'une anguille (marē) brûlée vive. D'après les habitants des îles Banks, une mère anguille donne naissance à une petite fille, qui adolescente se marie à un homme du village voisin. L'homme, profitant de l'absence de sa femme, brûle la mère anguille qui avait préalablement dicté ses vo- lontés à sa fille en prévision d'un tel drame. Sa fille enterre donc la tête de sa mère d'où émerge, quelques jours plus tard, un cocotier. La femme propose alors à son mari de boire l'eau du fruit. Après qu'il se soit réjoui de sa dégustation, elle lui fait remarquer qu'il s'agit en réalité de l'urine (mythe d'Ureparapara) ou du sang (Mota et Mota Lava) de sa mère serpent. Après ingestion de l'amande immature de la noix de coco, elle lui dit encore que ce sont les déjections (Ureparapara) ou la chair (Mota) de sa mère. Si ce mythe est largement répandu dans tout le Pacifique Sud, l'importance donnée à la mère, et en particulier la transmission de son sang et de sa chaire sous la forme d'eau de coco et d'amande, renforce l'existence du système matrilinéaire du nord du Vanuatu. Ainsi, les yeux et la bouche (le pore germinatif) sur les noix de coco rappellent à chacun le visage de la mère du cocotier ${ }^{22}$.

La création du cocotier a nécessité le sacrifice de la mère anguille par mort violente, le feu. Cette mort rôde désormais autour du couple cocotier/ homme. Ainsi, l'objet symbolisant la magie noire (bourre de coco, os brûlés et autres ingrédients) a été enterré non seulement sous le lit de l'homme visé mais aussi sous le cocotier qu'il avait planté. Les sorts du cocotier et de son planteur sont liés. De même, lorsqu'un contrat de mariage est conclu, deux cocotiers sont plantés côte à côte : la mort de l'un des deux présages du décès d'un des deux futurs mariés. Enfin, une catégorie de cocotier de forme spicata dénommée mōtō taktak est un cocotier de la mauvaise chance. Si l'on en a un dans son jardin, on mourra jeune. Si l'on remarque un jour que le nombre de fruits sur l'infrutescence correspond au nombre de ses frères et sœurs, et si le lendemain un des fruits est tombé, alors un enfant de la famille décèdera prochainement. Dans une histoire de Mota Lava, les folioles en mouvement, alors que le vent ne soufflait pas, indiquèrent à une femme se rendant au jardin, la mort de sa mère restée à la maison. De plus, sur cette même île, on raconte que si le héros commun au groupe des Banks, Qet, crée les êtres humains en sculptant des morceaux de bois, l'araignée Malawa annonça l'arrivée de la mort en enterrant ses sculptures humaines sur des nattes tressées de folioles de cocotier (Lanouguère-Bruneau, 2002) ${ }^{23}$. Ainsi, cocotiers et êtres humains sont liés par un contrat de vie

19. M. Rodman (1986) note le même procédé à Ambae où une noix de coco « chargée d'un maléfice » avait été enterrée sous le sentier menant aux maisons dont on voulait tuer le propriétaire.

20. Sur Mota Lava, la noix de coco peut également être utilisée comme châtiment : lorsqu'une personne manque de respect, même par inadvertance, à sa tante paternelle, elle doit manger une noix de coco souillée par de la terre (LanouguèreBruneau, 2002). À Ambae, les âmes des hommes morts s'abritent au sommet des arbres et des cocotiers (Rodman, 1995). 21. Au Cambodge, la vie d'une femme âgée est prolongée si sa mort et sa renaissance sont mimées par la destruction d'un corps de substitution, dont la tête est représentée par une noix de coco. De plus, chaque femme médium possède un " maître de naissance ", matérialisé par un objet, le kachom, dont l'élément principal est une noix de coco (Ang, 2005).

22. Sur Maewo, les fruits, dont les yeux pointaient jadis vers le sol, observaient les malheureux passants pour les tuer en se laissant tomber sur leur tête. Un jeune homme flécha les fruits pour les retourner et ainsi aujourd'hui ils ne font plus de mal aux êtres humains car aveugles. 
et de mort ; le cocotier, l'« alter-ego vivant " de l'homme (Giambelli, 1998 : 141), l'accompagne de sa naissance jusqu'à sa mort.

Pour que le destin d'une plante reflète celui d'un être humain, la plante doit présenter des qualités dignes du monde des hommes. Une histoire coutumière met justement en scène un cocotier intelligent qui a su résister à la tentation, et a choisi la meilleure stratégie de vie. Le cocotier qui marche mōtō vanvan et le bananier poussaient l'un à côté de l'autre quand le cocotier voulut aller sur les Reef Islands, pour relever le caractère sucré de charger son eau en sucre. Dans une autre version, le cocotier paye avec de la monnaie de coquillage un cochon hermaphrodite pour que celui-ci le fournisse en eau sucrée. En attendant son compagnon, le bananier alla chercher des coquillages de mer, du nom de rasēsēe, qu'il disposa autour de son pied. De chaque coquillage sortit des jeunes pousses. À son retour, le cocotier gronda le bananier car par cet acte il se condamnait à ne vivre qu'une seule année. Le cocotier, par contre, conserve ses fruits entre ses palmes et vit très longtemps. Ainsi, tous les six mois, le cocotier mōtō vanvan part se régénérer sur Reef Islands, et en son absence le cocotier ne porte pas de fruits ${ }^{24}$. De ce fait, le cocotier est un être doué de raison et d'intelligence. Mieux que le bananier qui meurt chaque année ou que le sagoutier qui s'éteint lors de son unique fructification, le cocotier, par sa patience et sa réflexion, a su se préserver de sa mort certaine en vivant plus longtemps et en produisant des fruits chaque mois. Lors de ses promenades sur Reef Island, le cocotier laisse ses attributs végétatifs ancrés dans le sol de l'île, mais possède la capacité de voyager vers d'autres lieux grâce à ses fruits. N'est-ce pas cette stratégie qui a permis au cocotier de diffuser ses semences dans tout le Pacifique?

Le cocotier est donc doué d'intelligence, et il présente les mêmes cycles biologiques qu'une femme. Fertile à sept ans, il rentrera lentement en " ménopause " vers 50 ans pour s'éteindre à plus de 70 ans. Durant sa vie, un organe fertile sera produit chaque mois. Après fécondation, chaque fleur mettra neuf à dix mois à se transformer en fruits suffisamment matures pour se détacher de leur mère et vivre une autre vie. Ses enfants souvent illégitimes, car d'origine paternelle inconnue, vivront non loin de leur mère ou prendront le large, voguant sur l'océan. Ces descriptions biologiques s'illustrent au mieux dans le mythe d'origine des gens de Lotawan, que l'on appelait anciennement les gens de Maligo, sur l'île de Mota (Vienne, 1984 : 87). Leur ancêtre commun est une petite fille qui tomba d'un cocotier comme une noix de coco. Plus qu'un visage, ses fruits peuvent être comparés à des seins de jeunes filles comme le montre le nom d'une des catégories nommées de cocotiers, le mōtō wēsusumalmàal " cocotier / poitrine de jeune fille ", dont les fruits sont ronds avec une petite pointe. L'eau des fruits de cocotier présente également une certaine analogie avec le lait maternel (À Bali, voir Giambelli, 1998), et dès sa naissance, l'enfant devait jadis avaler de l'eau de coco.

Le cocotier présente ainsi toutes les caractéristiques d'une femme, et plus précisément d'une mère. Comme d'autres en Indonésie (Engelhard, 1996), les habitants de Vanua Lava soulignent très justement la valeur reproductrice du cocotier lorsqu'ils offrent des fruits en germination à l'occasion d'un mariage ; la fertilité de la jeune mariée est honorée à travers ces offrandes. Depuis la naissance jusqu'à la mort, le cocotier est le « miroir » de ses habitants.

\section{Conclusion}

Ainsi le cocotier participe toujours à chaque étape de la vie des villageois, de la naissance au décès, à travers de nombreux usages quotidiens ou cérémoniaux, sous la forme de noix à boire, de lait incorporé dans des plats traditionnels, de fruits germés à planter, d'excipients pour des décoctions magiques, de matériel de construction et joue aussi le rôle principal dans des histoires coutumières.

Le fait que les catégories nommées de cocotiers les plus répandues à Vanua Lava sont des lexèmes immotivés, que ces mêmes catégories sont choisies préférentiellement pour nommer un cocotier lorsqu'il présente plusieurs caractéristiques, confirme l'ancienneté de ces catégories nommées. Pour ce qui concerne l'origine des autres noms, un grand nombre de catégories associées à un nom motivé décrivant la morphologie des fruits serait apparu plus récemment, avec la multiplication du nombre de cocotiers plantés. Sachant que les îles présentant les plus fortes diversités de catégories nommées sont surtout des îles à forte production de coprah, que ces mêmes îles présentent des noms décrivant très finement la noix et ses compartiments, nous incite à penser que le développement du coprah a eu une influence certaine sur la nomenclature locale en la complexifiant dans un registre descriptif. Les habitants du Vanuatu ont dû forger des termes nouveaux avec

23. Le cocotier peut également substituer la vie d'un cochon sur l'île de Pentecôte. Les chefs, pour monter en grade lors de la cérémonie Gonata, tuaient un seul cochon et demandaient à dix autres hommes de leur apporter dix fruits en germination. Le cochon et les dix noix de coco étaient offerts à un autre chef qui, ainsi, devenait le débiteur du donneur.

24. Au nord de l'île de Pentecōte, dans le village de Avatvotu, nous avons relevé le mēme type d'histoire. La permanence du cocotier est comparée à la fugacité du bananier qui chaque année meurt et doit se régénérer à partir de ses rejets ainsi qu'au sacrifice du sagoutier qui en fleurissant décède. 
des mots déjà présents dans leur langue. Ils ont su innover dans la cohérence d'un système préexistant dans toutes les îles pour l'ensemble des arbres à noix ; l'analyse de la dynamique du système de classification et de nomenclature du cocotier, nous renseigne ainsi sur le potentiel d'adaptation des hommes face à un environnement changeant (Friedberg, in Bonte, 1991).

Plantant plus d'arbres, les côtoyant plus intimement et plus souvent pour en extraire le coprah, le rapport qu'entretiennent les $\mathrm{Ni}$-Vanuatu avec leurs cocotiers, visible à travers ses systèmes de nomenclature, a été bouleversé par l'arrivée de cette économie. Le cocotier relève ainsi d'un statut « hybride » : sa valeur symbolique est un héritage du passé, mais son exploitation économique date de l'époque coloniale. Planté dans le village en petit nombre dans les temps pré-coloniaux, le cocotier était notamment valorisé pour sa rareté ; on ne le consommait pas tous les jours, réservant les rares fruits aux occasions festives ou d'échange. Aujourd'hui, alors que le cocotier-individu est devenu un élément de la cocoteraie, les anciens aussi bien que les jeunes vont parfois jusqu'à adopter une attitude de rejet face à cette plante " déchue " (Caillon, 2007). Ainsi, malgré les traditions classification, nomenclature, usages, histoires auxquelles il est lié, le cocotier est considéré désormais comme une plante "de Blancs».

\section{Remerciements}

Les informations rassemblées pour cette étude ethnobotanique nous ont été généreusement " offertes" par les cultivateurs de cocotiers. Nous remercions en particulier Louis Wōrvetel, Tomas Sakalmes, Hosea Waras, Banabas Manar, Dimas Wōrvetel, Edwin Tā̄atworlē, Elman, Henry Wiris, Hosea Qas, John Elman, Leynold, MacKenzie Tapē et Tony Romeo, ainsi que leur famille respective. L'hospitalité et l'aide ethnographique du chef Eli Field Malau et de sa femme Joana Rō Sōrōr Söm ont été particulièrement précieuses. Nous remercions aussi le Centre culturel du Vanuatu pour nous avoir facilité l'accès au terrain grâce à son réseau local d'assistants. Un grand merci à Sandrine Pavoine (MNHN) pour avoir plongé son regard de statisticienne dans ces données sociales. Elle a réalisé l'ensemble des figures et analyses sous $\mathrm{R}$. Le vurës a été vérifié par Catriona Hyslop-Malau et a pu être transcrit dans toute sa complexité grâce à la police fournie par Alexandre François. Les tests de dégustation et leurs analyses ont été menés grâce aux conseils avisés d'Alexia Prades. Merci à Jean-Pierre Labouisse pour ses relectures éclairées. Enfin, ce projet réalisé dans le cadre d'une thèse de géographie n'aurait pu exister sans le financement de la région Centre, du CIRAD et de l'IRD.

\section{BIBLIOGRAPHIE}

ANG Choulean, 2005. The human-nature relationship seen through animistic belief and ritual in Cambodia (consulté le 27 avril 2005, http://www.jpf.go.jp/e/culture/news/0412/ img/pdf/report08.pdf).

BARRAU Jacques, 1962. Les plantes alimentaires de l'Océanie : origines, distribution et usages, Marseille, Faculté des Sciences de Marseille, Annales du Musée colonial de Marseille, $63^{\mathrm{e}}$ à $69^{\mathrm{e}}$ années, $7^{\mathrm{e}}$ série, thèse de doctorat, $275 \mathrm{p}$.

Berlin B., D. E. Breedlove and P. H. Raven, 1974. The principles of Tzeltal plant classification, New York, London, Academic Press.

Bonnemaison Joël, 1996. Gens de pirogue et gens de la terre, Paris, Éditions de l'orstom.

Bonte Pierre et Michel Izard (éds), 1991. Dictionnaire de l'ethnologie et de l'anthropologie. Paris, Quadrige/PuF.

Caillon Sophie, 2004. Kokonas mo taro blong Vanuatu: wan katalog, Orléans, IRD.

—, 2007. Arbre d'antan, arbre "des Blancs". Évolution de la valeur sociale des cocotiers et de leur espace à Vanua Lava (Vanuatu), Géographie et Culture 63, pp. 87-104.

Caillon Sophie et Virginie Lanouguère-BruNEAU, 2005. Gestion de l'agrobiodiversité dans un village de Vanua Lava (Vanuatu) : stratégies de sélection et enjeux sociaux, Journal de la Société des Océanistes 120-121, pp. 129-148.

Dubois J., M. Giacomo, C. Marcellesi, J.-B. Marcellesi et J.-P. Mével, 1994. Dictionnaire de linguistique et des sciences du langage, édité par c. T. d. Français, Paris, Larousse.

Friedberg Claudine, 1968. Les méthodes d'enquête en ethnobotanique. Comment mettre en évidence les taxonomies indigènes ?, Journal d'Agriculture traditionnelle et de Botanique appliquée 15 (7-8), pp. 297-324.

—, 1997. Diversité, ordre et unité dans les savoirs populaires, Natures, Sciences et Sociétés 5 (1), pp. 5-17.

Giambelli Rodolfo A., 1998. The coconut, the body and the human being. Metaphors of life and growth in Nusa Penida and Bali, in L. Rival (ed.), The social life of trees. Anthropological perspectives on tree symbolism, Oxford, New York, Berg, pp. 133-158.

Grenand Françoise, 2001-2002. Stratégies de nomination des plantes cultivées dans une société tupi-guarani, les Wayãpi, Amerindia 26/27, pp. 209-247. 
Harlan Jack R., 1987 [1975]. Les plantes cultivées et l'homme, Paris, Presses Universitaires de France.

Haudricourt André G., 1964. Nature et culture dans la civilisation de l'igname : l'origine des clones et des clans, L'Homme 4 (1), pp. 93-104.

Hess Sabine, 2009. Person and Place. Ideas, Ideals and the Practice of Sociality on Vanua Lava, Vanuatu, London, Berghahn.

LABOuIsse Jean-Pierre, 2004. Systèmes agraires et économie du cocotier au Vanuatu : historique et perspectives, Journal de la Société des Océanistes 118, pp. 11-33.

Labouisse Jean-Pierre et Sophie Caillon, 2001. Une approche de la conservation in situ par l'étude d'un système semencier informel : cas du cocotier au Vanuatu (Pacifique Sud), OCL 8 (5), pp. 534-539.

Labouisse Jean-Pierre, Tiata Sileye, Jean-Paul Morin, Chantal Hamelin, Luc Baudouin, Roland Bourdeix et André Rouzière, 2004. Coconut (Cocos nucifera L.) genetic improvement in Vanuatu: overview of research achievements from 1962 to 2002. Part I: Improvement of Vanuatu Tall by mass selection, OCL 11 (4-5), pp. 354-361.

—, 2005. Coconut (Cocos nucifera L.) genetic improvement in Vanuatu : overview of research achievements from 1962 to 2002. Part 2: Improvement of the Vanuatu Tall by hybridization, OCL 12 (2), pp. 170-179.

Lanouguère-Bruneau Virginie, 2002. Le corps de l'igname et le sang de la noix de coco. Le système social ancien de Mota Lava (îles Banks-Vanuatu), thèse en Anthropologie sociale, Paris, EHEss, 453 p.

Malau Catriona (2011-05-05) Dictionary of Vurës (http://paradisec.org.au/VurësLexicon/ index-english/main.htm)
Martin M.-A. 1975. L'ethnobotanique, science per se? À propos d'un livre de B. Berlin "The principles of Tzeltal plant classification", Journal d'Agriculture tropicale et de Botanique appliquée XXII (7-8-9), pp. 237-276.

Mialet-Serra Isabelle et Philippe Taler, 2003. Les réserves carbonées chez le cocotier, le palmier à huile, l'hévéa et le manguier : origines, dynamiques et conséquences pour la gestion des plantations - Premier compte-rendu annuel technique et financier d'ATP/CIRAD, Montpellier, CIRAD-AMIS, $50 \mathrm{p}$.

Ohler J. G., 1984. Coconut, tree of life, Rome, FaO.

Petard Paul, 1986. Quelques plantes utiles de Polynésie Française et Raau Tahiti, Papeete, Éditions Haere Po No Tahiti.

R-Development-Core-Team, 2008. R: A Language and Environment for Statistical Computing, Vienna, Austria, R Foundation for Statistical Computing.

Rodman Margaret, 1986. Enracinement de l'identité : La tenure foncière à Longana, Vanuatu Culture 6 (2), pp. 3-13.

-, 1995. Breathing spaces : customary land tenure in Vanuatu, in G. R. Ward et E. Kingdon (eds), Land, custom and practise in the South Pacific, Cambridge, Cambridge University Press, pp. 65-108.

Sauvageot François et Catherine DacreMONT, 1995. L'évaluation sensorielle à la portée de tous ou les premiers pas en évaluation sensorielle, Dijon, ENsBana, Université de Bourgogne.

SPEISER Felix, 1996 [1923]. Ethnology of Vanuatu. An early twentieth century study, Bathurst, Australia, Crawford House Publishing Pty Ldt.

Vienne Bernard, 1984. Gens de Motlav. Idéologie et pratique sociale en Mélanésie, Paris, Société des Océanistes, Publications de la Sdo 42. 
